JULY 2011

Preprint typeset using $\mathrm{AT}_{\mathrm{E} X} \mathrm{X}$ style emulateapj v. 11/10/09

\title{
OBSERVATIONAL CONSTRAINTS ON RED AND BLUE HELIUM BURNING SEQUENCES
}

\author{
Kristen B. W. McQuinn ${ }^{1}$ Evan D. Skillman $^{1}$ Julianne J. Dalcanton $^{2}$, Andrew E. Dolphin ${ }^{3}$, Jon Holtzman $^{4}$, \\ Daniel R. Weisz ${ }^{2}$, \& Benjamin F. Williams ${ }^{2}$ \\ July 2011
}

\begin{abstract}
We derive the optical luminosity, colors, and ratios of the blue and red helium burning (HeB) stellar populations from archival Hubble Space Telescope observations of nineteen starburst dwarf galaxies and compare them with theoretical isochrones from Padova stellar evolution models across metallicities from $Z=0.001$ to 0.009 . We find that the observational data and the theoretical isochrones for both blue and red HeB populations overlap in optical luminosities and colors and the observed and predicted blue to red $\mathrm{HeB}$ ratios agree for stars older than $50 \mathrm{Myr}$ over the time bins studied. These findings confirm the usefulness of applying isochrones to interpret observations of $\mathrm{HeB}$ populations. However, there are significant differences, especially for the red HeB population. Specifically we find: (1) offsets in color between the observations and theoretical isochrones of order $0.15 \mathrm{mag}$ ( $0.5 \mathrm{mag})$ for the blue (red) HeB populations brighter than $\mathrm{M}_{V} \sim-4 \mathrm{mag}$, which cannot be solely due to differential extinction; (2) blue HeB stars fainter than $\mathrm{M}_{V} \sim-3 \mathrm{mag}$ are bluer than predicted; (3) the slope of the red HeB sequence is shallower than predicted by a factor of $\sim 3$; and (4) the models overpredict the ratio of the most luminous blue to red HeB stars corresponding to ages $\lesssim 50$ Myr. Additionally, we find that for the more metal-rich galaxies in our sample $\left(Z \gtrsim 0.5 \mathrm{Z}_{\odot}\right)$ the red HeB stars overlap with the red giant branch stars in the color magnitude diagrams, thus reducing their usefulness as indicators of star formation for ages $\gtrsim 100 \mathrm{Myr}$.

Subject headings: galaxies: dwarf — galaxies: evolution — galaxies: individual (Antlia dwarf, ESO 154-023, UGC 4483, UGC 6456, UGC 9128, NGC 625, NGC 784, NGC 1569, NGC 2366, NGC 4068, NGC 4163, NGC 4214, NGC 4449, NGC 5253, NGC 6789, NGC 6822, IC 4662, DDO 165, Holmberg II) - galaxies: starburst
\end{abstract}

\section{HEB STARS IN ISOCHRONES AND OBSERVATIONAL CMDS}

Theoretical stellar evolutionary isochrones predict the paths that stars will traverse in a color magnitude diagram (CMD) as the stars age and evolve. Isochrone libraries span a large range of stellar masses and metallicities (e.g., VandenBerg et al. 2000; Bertelli et al. 1994; Lejeune \& Schaerer 2001; Yi et al. 2001; Pietrinferni et al. 2004; Dotter et al. 2008; Marigo et al. 2008, and references therein). They are widely used to aid in the interpretation of CMDs constructed from stellar populations observed in clusters and galaxies including measuring the star formation histories (SFHs) of galaxies (e.g., Tolstov \& Saha 1996; Aparicio et al. 1996; Dolphin 2002; Harris \& Zaritsky 2001). Isochrones have also aided both in understanding of the integrated properties of stellar populations (e.g., Bruzual \& Charlot 2003; Leitherer et al. 2010, and references therein) and in quantifying the chemical and spectral evolution of galaxies. Current theoretical isochrones use radiative opacities (Bertelli et al. 1994) and modified combinations of convective core and convective en-

\footnotetext{
${ }^{1}$ Department of Astronomy, School of Physics and Astronomy, 116 Church Street, S.E., University of Minnesota, Minneapolis, MN 55455,kmcquinn@astro.umn.edu

2 Department of Astronomy, Box 351580, University of Washington, Seattle, WA 98195

${ }^{3}$ Raytheon Company, 1151 E. Hermans Road, Tucson, AZ 85706

${ }^{4}$ Department of Astronomy, New Mexico State University, Box 30001-Department 4500, 1320 Frenger Street, Las Cruces, NM 88003
}

velope overshooting (Alongi et al. 1993; Bressan et al. 1993) calibrated to the different wavelengths and filters commonly used in observations (Girardi et al. 2004).

In this paper we focus on luminous, core helium burning $(\mathrm{HeB})$ stars. These stars have initial masses between $\simeq 2-15 \mathrm{M}_{\odot}$, and evolve off the main sequence (MS) between ages of $\simeq 5-1000$ Myr into HeB stars before evolving further into thermally pulsating asymptotic giant branch (TP-AGB) stars or exploding as supernovae. Specifically, current models show stars in this initial mass range will leave the MS burning helium in their cores (e.g., Gallart et al. 2005, and references therein), becoming more luminous and redder in color, and will populate the red $\mathrm{HeB}(\mathrm{RHeB})$ branch in a CMD. The RHeB stars will traverse the CMD toward bluer colors, making the "blue loop", and populate the blue $\mathrm{HeB}(\mathrm{BHeB})$ branch. A comparison of the predicted characteristics of HeB stars with observations can provide important constraints for the stellar evolution models, particularly with high-fidelity photometric measurements now achievable from space-based telescope observations.

Based on the current Padova models (for a comprehensive review, see Chiosi et al. 1992), both the RHeB and $\mathrm{BHeB}$ phases of evolution occupy a space in an optical CMD which is uniquely dependent on the age of the star. While other phases of evolution have either an age degeneracy or an age-metallicity degeneracy in their CMD location, the one-to-one age-luminosity relation of $\mathrm{HeB}$ stars affords an opportunity to not only separate stars in this stage of evolution from other populations, but to also age date the HeB population in an observed 
CMD directly from isochrones (e.g., Dohm-Palmer et al. 1997). HeB stars spend more time at or near the extreme red and blue colors than at intermediate colors as $\mathrm{HeB}$ stars evolve rapidly from the $\mathrm{RHeB}$ to the $\mathrm{BHeB}$ phase. The luminosities and colors around these extrema define the RHeB and $\mathrm{BHeB}$ sequences observationally and are readily identifiable in an observed CMD. The equivalent red and blue extrema are also easily identified in theoretical isochrones. Thus, the HeB populations provide an excellent opportunity to test the predictions of theoretical isochrones on an individual stage of stellar evolution. In addition, the ratio of $\mathrm{BHeB}$ stars to $\mathrm{RHeB}$ stars, or $B / R$ ratio, is now measurable for resolved stellar populations. The $\mathrm{B} / \mathrm{R}$ ratio is dependent on convection, mass-loss, and stellar rotation, and thus also provides important diagnostics for stellar evolutionary models (e.g., Dohm-Palmer \& Skillman 2002, and references therein).

To illustrate the importance of $\mathrm{HeB}$ stars for constraining stellar evolution, one can consider the historical development of stellar models with an initial mass between $\simeq 2-15 \mathrm{M}_{\odot}$. For these stars, it was originally assumed that the sole opacity source was electron scattering, which was later revised to include bound-free absorption (Havashi \& Cameron 1962; Stothers 1966). Other studies assumed the unstable part of the stellar envelope of massive stars was fully convective (Iben 1966) instead of semi-convective. Both the opacity assumption and convection assumption predicted that massive stars $\left(\mathrm{M}>15 \mathrm{M}_{\odot}\right)$ would evolve off the MS directly into blue supergiants or BHeB stars. Red supergiants were thought to have a different origin and were explained as a later phase of evolution of stars with carbon-burning cores (Hayashi et al. 1962).

The subsequent addition of bound-free absorption and semi-convective envelopes in the stellar evolution models (Stothers \& Chin 1968) changed our understanding of the evolutionary paths of the massive stars; i.e., they correctly predicted that both blue and red supergiants are core $\mathrm{HeB}$ stars, and that MS stars evolve first into RHeB stars and then into $\mathrm{BHeB}$ stars. With the blue and red supergiants both identified as core HeB stars, the RHeB stars were predicted to traverse the CMD as they evolve into $\mathrm{BHeB}$ stars when the mass fraction of hydrogen shell burning increased and the efficacy of cooling by boundfree absorption was reduced. Revisions of the triple- $\alpha$ nuclear reaction rate induced the formation of a blue loop for all but the most massive stars (Austin et al. 1971). Further, the blue loop was lengthened due to changes in the cross-section of the ${ }^{12} \mathrm{C}(\alpha, \gamma){ }^{16} \mathrm{O}$ nuclear reaction (see $\S 6.3$ Gallart et al. 2005, and references therein).

The predicted ratio of the blue to red $(\mathrm{B} / \mathrm{R}) \mathrm{HeB}$ stars (i.e., predictions of the evolutionary time spent in each core HeB phase) was found to depend on the prescription for convection, with the Ledoux criterion (Ledoux 1947; Sakashita et al. 1959; Stothers \& Chin 1975, and references therein) better reproducing the general trends in the distribution of blue and red supergiants than the Schwarzschild criterion (Stothers \& Chin 1976, and references therein). The inclusion of downward convective envelope overshooting more consistently predicted the occurrence of blue loop stars (Stothers \& Chin 1991) favoring these models over those including convective core overshooting, which under-predicted the blue colors of the BHeB stars (Bertelli et al. 1985; Stothers \& Chin
1992) or over-predicted the luminosity of the BHeB stars (Alongi et al. 1993, and references therein).

In spite of these successes, discrepancies in the models of the HeB stars remained. Further study of RHeB stars revealed that the stellar evolution models that correctly predicted the number of red supergiants at low metallicities over-predicted the corresponding number of $\mathrm{RHeB}$ stars in solar metallicity systems and vice-versa (e.g., Langer \& Maeder 1995, and references therein). In response, stellar rotation was added to the models, increasing internal mixing and causing a larger He-core. The smaller associated intermediate convective zones permitted the stellar radius of rotating stars to inflate during the He-burning phase, favoring the evolution of stars towards red supergiants (Maeder \& Mevnet 2001). This increased the number of $\mathrm{RHeB}$ stars, thus producing $\mathrm{B} / \mathrm{R}$ ratios more consistent with low metallicity observations. However, accurately measuring the $B / R$ ratio from ground based observations of this era was difficult as the lower photometric accuracy blended the MS and $\mathrm{BHeB}$ stars into the "blue plume" of an optical CMD.

Using observations from the Hubble Space Telescope (HST) archive, we expand on the work by Dohm-Palmer \& Skillman (2002) for Sextans A and measure the luminosity, color, and ratios of the optically resolved BHeB and RHeB stellar sequences in the CMDs of nineteen starburst dwarf galaxies. Starburst galaxies were selected as they are known to contain significant populations of $\mathrm{HeB}$ due to their recent and/or ongoing star formation. The reduction in uncertainty due to the large number of $\mathrm{HeB}$ stars is somewhat offset by the associated increase in uncertainty due to the higher crowding and differential extinction typical in regions of high star formation (Boquien et al. 2009). The galaxy sample spans a range of chemical composition from $Z=0.0007$ to 0.0093 allowing us to test isochrones of metallicities up to $\simeq 0.5 \mathrm{Z}_{\odot}$. We compare the measured sequences and the $\mathrm{B} / \mathrm{R}$ ratios to those derived from Padova stellar evolution isochrones. The Padova isochrones were selected for comparison because they are currently the only isochrones with sufficient metallicity and high mass star coverage, and, conveniently, the isochrones are calibrated to the HST filters used for the observations.

In 92 we present the theoretical isochrones and describe how the predicted $\mathrm{BHeB}$ and $\mathrm{RHeB}$ sequences were identified. In 43 , we describe the galaxy sample and how the sequences were identified from the observational data. In 44 we compare the theoretical and empirical results. We present a discussion of the impact of these results in $\$ 5$ and our conclusions in $\$ 6$.

\section{MEASURING THE BHEB AND RHEB SEQUENCES OF PADOVA ISOCHRONES}

The predicted luminosity, color, and ratio of $\mathrm{HeB}$ stars of different ages (i.e., masses) can be discerned from theoretical isochrones. We use the theoretical isochrones of Girardi et al. (2000) for stars with masses $\leq 7 \mathrm{M}_{\odot}$ with improved treatment of the asymptotic giant branch (AGB) stars added by Marigo et al. (2008). At higher masses $\left(M>7 M_{\odot}\right)$, we use the isochrones of Bertelli et al. (1994). Collectively, this set of isochrones is known as the Padova stellar evolution isochrones. The Padova isochrones (hereafter "isochrones") were selected for comparison with observations because they met three 
criteria. First, the isochrones are available for the range in metallicities of the galaxy sample. Second, the Padova stellar evolution models include high mass stars that populate the blue and red $\mathrm{HeB}$ sequences. Third, the isochrones provide predicted magnitudes calibrated to the HST filters and are thus directly comparable to the observational data used in this study. The basic outputs of the stellar evolution models are surface luminosity and effective temperatures $\left(T_{e f f}\right)$ of stars. To allow comparison with observed photometric data, these quantities are converted to magnitudes and color for various bandpasses using bolometric corrections and $T_{\text {ef }} f^{-}$ color relations from stellar atmosphere models, namely the ATLAS9 stellar spectra library (Castelli \& Kurucz 2003). For a complete description of the stellar atmosphere libraries used, see Girardi et al. (2008) and references therein. We chose not to compare the data to the BaSTI models (Pietrinferni et al. 2004) as the isochrones do not cover include the higher mass HeB stars. Similarly, we chose not to use the updated Geneva models (e.g., Meynet \& Maeder 1997) as the isochrones cover only metallicities of $Z=0.00001,0.004$, or solar abundance.

Isochrones of seven different chemical compositions were selected with $Z$ ranging from $0.001-0.019$. The six lowest metallicity isochrones approximately match the metallicities of our galaxy sample as listed in Table 1. The highest metallicity is equivalent to solar abundance, and is included for comparison purposes. We generate these seven sets of isochrones for three different permutations of HST instruments and filters, listed in Table 2 , While our data were not obtained with the bluer HST filter F475W, we include predictions for the F475W and F814W combination for comparison purposes and future reference. We chose not to use circumstellar dust models (Bressan et al. 1998; Groenewegen 2006) which incorporate mass loss to asymptotic giant branch (AGB) and post-AGB stars as the dust models impact the signatures of AGB stars and not HeB stars.

In Figure 1, we present an example of the theoretical isochrones used in our analysis. The isochrones for a single metallicity of 0.003 are plotted for one set of Vand I-band filters, for stellar ages from $\log t=7.0-$ 9.0 at increments of $\log \delta \mathrm{t}=0.15$. Each line traces the CMD location of stars of the same age but with different masses, thus showing the position of main sequence (MS) stars, red and blue HeB stars, and their evolution off the CMD at the end of the life cycle. We do not show isochrones for ages greater than $1 \mathrm{Gyr}$, i.e., for lower mass stars $\left(\lesssim 2 \mathrm{M}_{\odot}\right)$ that would evolve onto the red giant branch (RGB) without becoming $\mathrm{HeB}$ stars. Note that the $\mathrm{BHeB}$ stars have values of $M_{V} \sim 2$ mag brighter than MS stars of equal mass.

$\mathrm{HeB}$ stars will be found at colors and magnitudes which approximately correspond to the red and blue extrema of the isochrones, corresponding to the beginning of the core $\mathrm{HeB}$ stars and the maximum temperature of the $\mathrm{BHeB}$ phase. As $\mathrm{HeB}$ stars spend more time near these extrema than at intermediate colors and magnitudes, the HeB stars create two loci of points or "sequences" in the CMD (see \$3.1 Figure 5, for example). However, when weighted by the duration the stars spend in the $\mathrm{HeB}$ phases, these sequences do not necessarily correspond exactly to the extrema. It is the luminosity and color of the

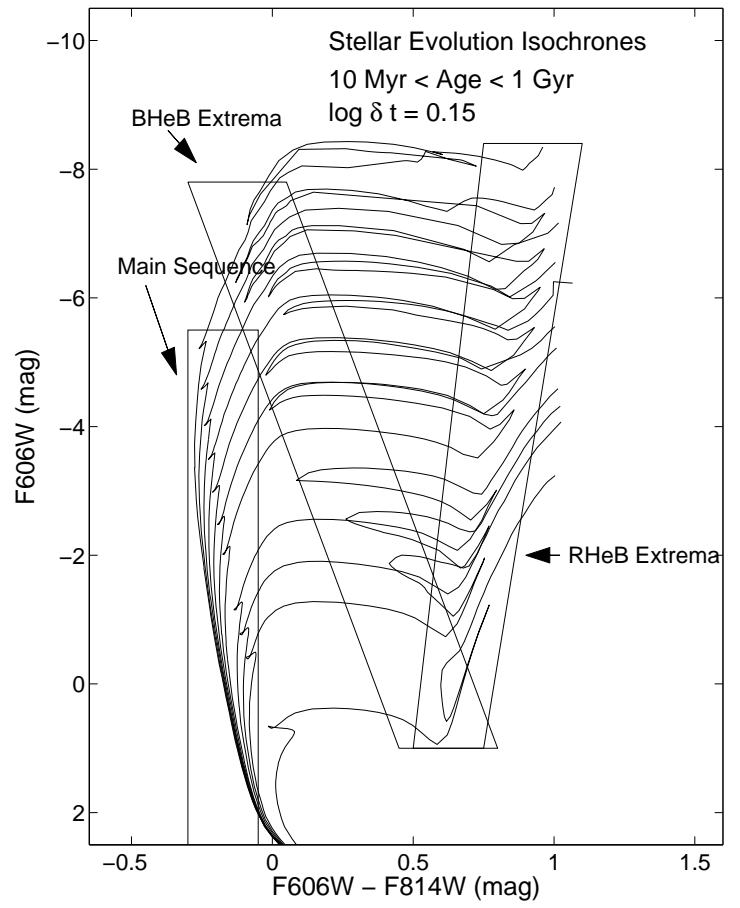

Fig. 1. -0.003 isochrones for stars with ages between $\log \mathrm{t}=$ 7.0 to 9.0 at increments of $\log \delta \mathrm{t}=0.15$ for the $\mathrm{F} 606 \mathrm{~W}$ and F14W filters. Three evolutionary sequences are identified in the figure: the main sequence of stars, the red extrema of the $\mathrm{HeB}$ stars occurring after the stars leave the main sequence, and finally the blue extrema of the $\mathrm{HeB}$ stars reached after the $\mathrm{RHeB}$ stars traverse back toward bluer colors in the CMD. The isochrones are cut-off after the BHeB stage of evolution to avoid confusion in the plot.

actual blue and red HeB sequences (i.e., not the extrema of the isochrones) that correspond to the sequences in an observed CMD and thus of interest here. Thus, to determine the exact location of the sequences, we generated synthetic CMDs using seven different metallicity isochrones, assuming a Salpeter initial mass function (Salpeter 1955) for two different V and I filter combinations. An example synthetic CMD is shown in Figure 2 for the isochrone $Z=0.003$ and the $\mathrm{F} 606 \mathrm{~W}$ and $\mathrm{F} 814 \mathrm{~W}$ filter combination.

To determine the locations of these sequences in the CMDs, we binned the stars in the synthetic CMD in color over nine one-magnitude wide luminosity intervals. We present an example histogram of a one-magnitude slice in Figure 3. The three peaks in each histogram correspond to the MS stars, the $\mathrm{BHeB}$ stars, and the $\mathrm{RHeB}$ stars. The theoretical $\mathrm{BHeB}$ sequence was determined by the colors of $\mathrm{BHeB}$ distribution peaks at each of the nine one-magnitude intervals in luminosity. The RHeB sequence was determined using the same method, but over a smaller magnitude range as $\mathrm{RHeB}$ stars do not reach the same high luminosities as the $\mathrm{BHeB}$ stars at optical wavelengths. We plot these sequences as solid lines on the synthetic CMD in Figure 2 and compare them to the location of the blue and red extrema shown as the dashed lines. As expected, the histogram-determined sequences lay on the centers of the blue and red $\mathrm{HeB}$ stellar distributions, slightly offset from the extrema.

In Figure 4, we present the theoretical predictions for the blue and red $\mathrm{HeB}$ sequences for seven metallicities and different optical filter combinations. The top pan- 


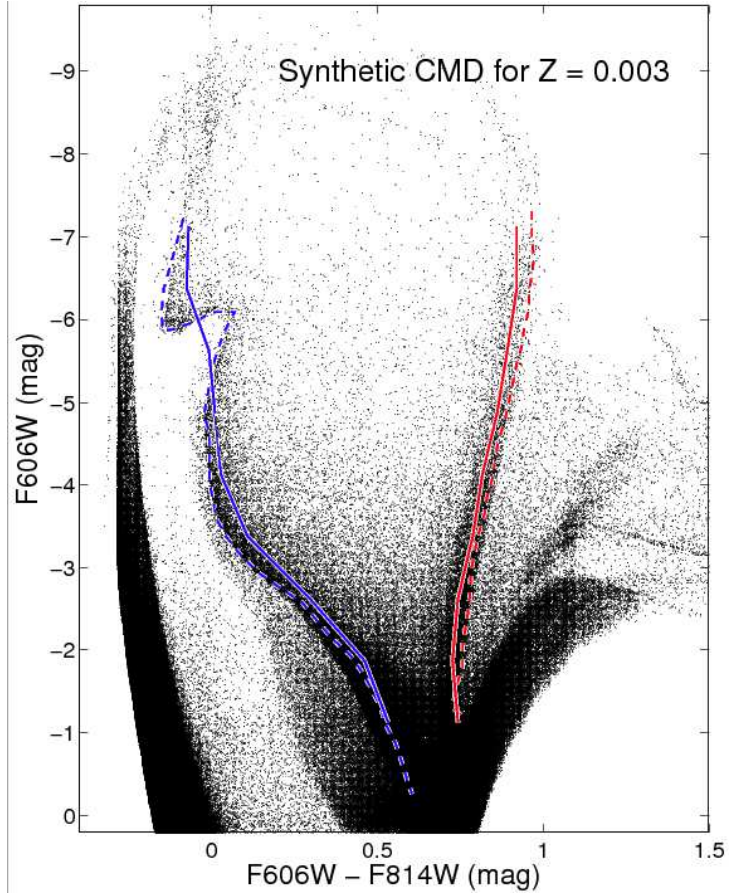

FIG. 2.- A synthetic CMD generated for a metallicity of $Z=$ 0.003. The model assumes increasing SFRs at recent times to fully populated the CMD with higher luminosity, higher mass stars. The two dashed lines represent the blue and red extrema of the $\mathrm{HeB}$ tracks in the isochrones. The blue and red $\mathrm{HeB}$ stars spend most of their time approaching the extrema, thus the HeB stars in the synthetic CMD lie near the isochrone predicted sequences but with some distribution in color. We determine the luminosity and color of the HeB sequences from the synthetic CMDs, given that the observed HeB sequences will 'pile-up' in a similar fashion as they approach the extreme colors of the blue loop. The adopted sequences are shown as solid lines.

els show the blue (left) and red (right) HeB track for the F606W and F814W filters for the HST Advanced Camera for Surveys (ACS; Ford et al. 1998). The colored lines represent different metallicities. The lowest metallicity ( $Z=0.001$; blue line) has the overall bluest colors for both the blue and red HeB sequences. The highest metallicity $(Z=0.019$ or solar abundance; black line) has the reddest colors for the blue and red $\mathrm{HeB}$ sequences. The middle panels of Figure 4 show the HeB tracks for F555W and F814W filters for the HST Wide Field Planetary Camera 2 (WFPC2; Holtzman et al. 1995) instrument. The HeB sequence generated from isochrones for the ACS F555W and F814W filters were equivalent to those generated for the equivalent WFPC2 filters. We therefore use the isochrone set from the WFPC2 filters for comparison with observations from both instruments and refer to the isochrone set by filter, not by instrument. The bottom panels of Figure 4 show the HeB tracks generated for F475W and F814W filters for the HST ACS instrument. The isochrones using this longer color baseline cover a larger range in color in the CMDs.

\section{MEASURING THE BHEB AND RHEB SEQUENCES IN THE OBSERVED CMDS}

\subsection{The Galaxy Sample and Their Optically Resolved Stellar Populations}

The galaxy sample was comprised of nineteen, nearby starburst dwarf galaxies, previously studied by McQuinn et al. (2010b, and references therein). All of

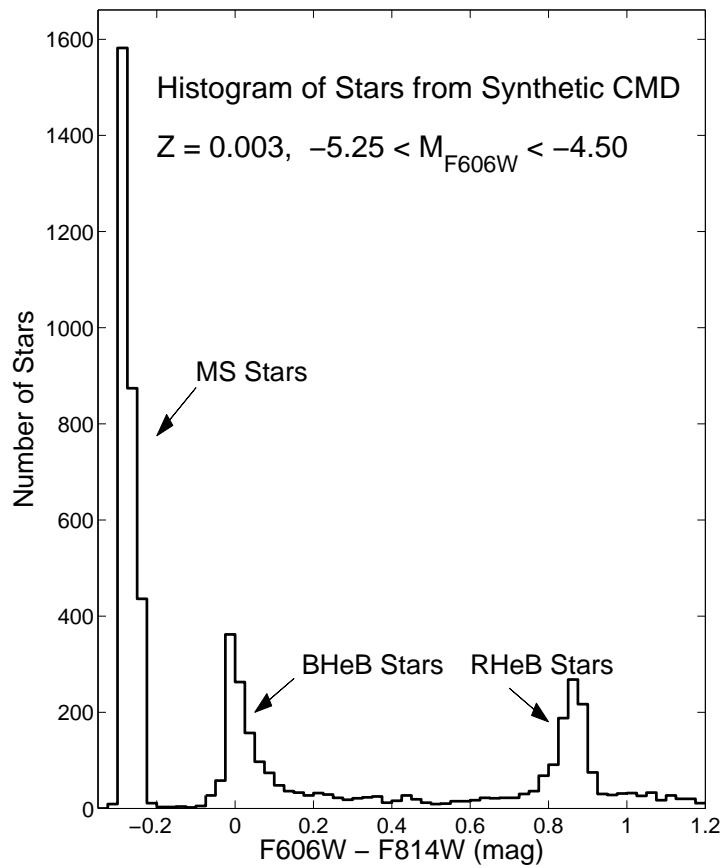

FIG. 3.- An example histogram of stars from the synthetic CMD in Figure 2 between the luminosity range $=5.25<\mathrm{M}_{V}<-4.50$. The first peak in the histogram is populated by MS stars, the second peak by BHeB stars, and the final peak by RHeB stars. The color separation between the difference sequences is evident in the figure allowing the luminosity and color of the HeB sequences to be unambiguously identified. We chose the peak of the blue and red $\mathrm{HeB}$ histogram peaks to define the sequences.
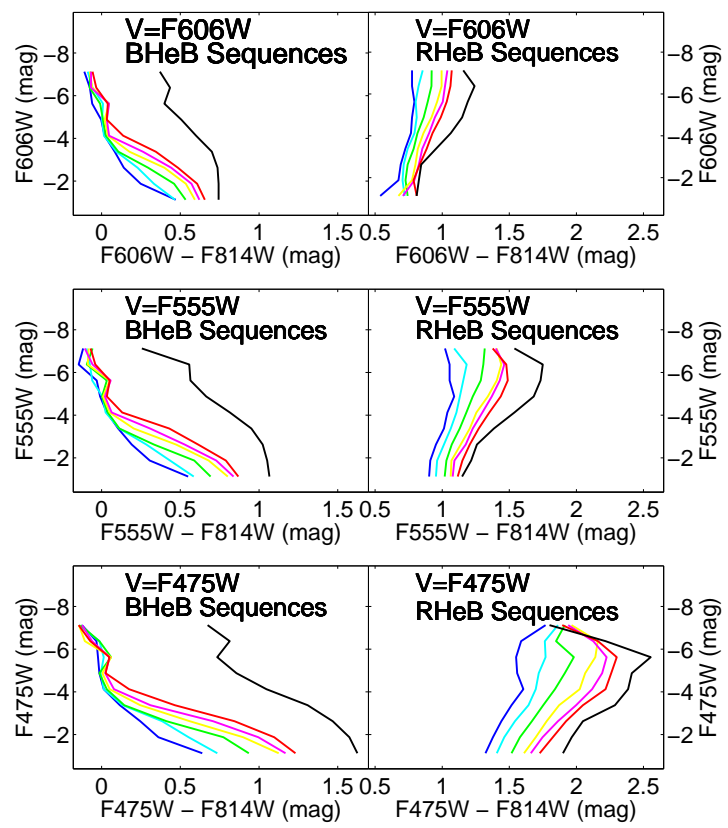

FIG. 4.- Theoretical BHeB and RHeB sequences for three filter combinations. The colors represent different isochrone metallicities matching the colors and metallicity range of the observed galaxy sample shown in Figure 8 Color key: blue is $Z=0.001$, cyan is $Z=0.002$, green is $Z=0.003$, yellow is $Z=0.005$, magenta is $Z=0.006$, red is $Z=0.009$, black is $Z=0.019$. The black sequence represents solar abundances and is added for comparison purposes. Note that the small shifts in the V filter between F606W, F555W, and F475W, causes a measurable shift in the colors of the sequences. 
the galaxies have high quality HST multi-color optical imaging taken with either ACS or WFPC2, previously reduced and photometered as described in McQuinn et al. $(2010 \mathrm{a}, \mathrm{b})$. This sample forms an ideal set for identifying $\mathrm{HeB}$ sequences as the recent rates of star formation are sufficiently high such that the blue and red HeB branches of the observed CMD are well-populated. While there is some associated increase in uncertainty in a starburst galaxy sample due to the higher crowding typical in regions of high star formation, most of our sample is comprised of low metallicity dwarf galaxies that have relatively small amounts of differential extinction. Thus, our choice in galaxies reduces, although does not eliminate, the effects of differential extinction and the potential for blending of $\mathrm{MS}$ and $\mathrm{BHeB}$ stars in a $\mathrm{CMD}$, in spite of our sample's high recent star formation rates (SFRs) and high gas content.

Individual metallicities for the sample are listed in Table 1 . The metallicities are derived either from $\mathrm{H}$ II region abundances taken from the literature or from estimates derived from the luminosity-metallicity relation (Zaritsky et al. 1994; Tremonti et al. 2004; Lee et al. 2006), as noted. The present-day metallicities are likely to match those of the $\mathrm{HeB}$ populations, given that no significant chemical evolution is expected or observed over the last $\sim 250$ Myr. Studies of the oxygen abundances in $\mathrm{H}$ II regions show the gas is well mixed in dwarf galaxies on short timescales (Skillman et al. 1989; Kobulnicky \& Skillman 1997b; Lee et al. 2006), thus any variations in metallicity in the young stellar populations within an individual galaxy should be negligible.

\subsection{Correcting the Observations for Reddening}

The Galactic foreground and internal extinction in the $\mathrm{V}$ filter was estimated through fitting the observed CMD to synthetic CMDs as described in McQuinn et al. (2009, 2010a). Briefly, we reconstructed the SFHs by finding the best fit synthetic CMD to an observed CMD allowing the distance and the average uniform foreground extinction to be included as parameters. A differential extinction parameter was added in cases where the observed CMD showed signs of internal extinction, typically manifested as broadening of the MS beyond what is expected from photometric uncertainties. We list our total estimated extinction, including both foreground and internal extinction values, in Table 1 and compare them with the foreground extinction estimates from the dust maps of Schlegel et al. (1998). In sixteen cases, our estimates are within $0.1 \mathrm{mag}$ of those from Schlegel et al. (1998). In three cases, our estimates of the total extinction are greater than those of Schlegel et al. (1998) by $0.19-0.32 \mathrm{mag}$, indicating the presence of internal extinction. Including estimates of internal extinction optimizes the chance for the models to fit the data, thus any significant discrepancies between the models and data in these three systems is more likely attributable to model parameters.

The extinction for the F814W filter was determined by extrapolating our estimated $A_{V}$ values using the York Extinction Solver tool (McCall 2004) which applied the reddening law of Cardelli et al. (1989) with $\mathrm{R}=3.1$. These $A_{F 814 W}$ values are also listed in Table 1. We adopt our estimated constant values for de-reddening and account for the extinction by subtracting our estimated
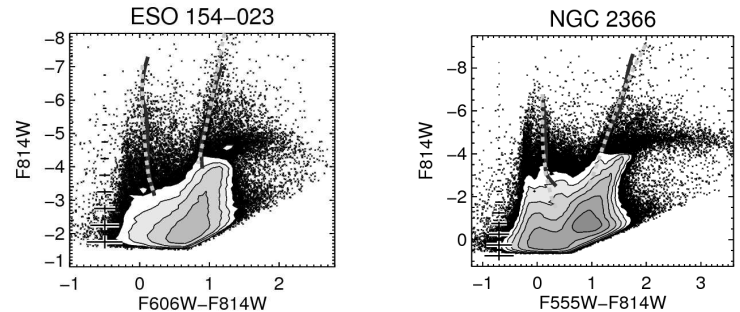

FIG. 5.- Optical CMDs of a galaxy reaching a photometric depth representative of the sample (top panel; ESO154-023), and of a galaxy reaching one of the deepest photometric depths of the sample (bottom panel; NGC 2366). The dashed lighter lines denote the blue and red $\mathrm{HeB}$ sequences selecting by eye on the CMD. The solid darker lines are the same HeB sequences selected using histogram plots of stars in one-magnitude intervals as shown in Figure 6] The blue and red HeB sequences selected with the two methods are in good agreement with each other.

$A_{V}$ values from the stellar photometry and our estimated $A_{V}-A_{F 814 W}$ values from the measured $V-I$ stellar colors. The extinction reported in Table 1 does not account for the potential presence of differential extinction that may be located around the youngest stars; we consider the effects on the observed blue and red HeB sequences of such potentially non-uniform extinction in 4.1 and 4.2

\subsection{Isolating the HeB Populations}

The $\mathrm{BHeB}$ stars populate a region of the optical CMD approximately parallel in magnitude to the MS stars. As long as the photometry are of high quality, the photometric errors at the brighter magnitudes of the $\mathrm{HeB}$ stars are smaller than the inherent separation in color between the two populations and the two distinct populations are easily separated in color-magnitude space. There is some contamination of the $\mathrm{BHeB}$ sequence as stars leaving the MS and evolving into RHeB stars pass through this loci of points. However, these stars spend very little time making the transition and are conservatively estimated to produce a contamination of less than $\sim 10 \%$ (Dohm-Palmer \& Skillman 2002). Likewise, the $\mathrm{RHeB}$ stars form a sequence that lies blue-ward of the RGB.

We take two approaches to selecting the loci of both the $\mathrm{BHeB}$ and the RHeB stars in the observational CMDs to ensure the most robust selection of $\mathrm{HeB}$ stars with the smallest contamination from MS or RGB stars. Here, we describe the two methods for selecting the BHeB stars in the CMDs; these same methods are applied in selecting the RHeB stars. First, the center of the BHeB stellar distribution is marked in the CMD, shown as a dashed line in the CMDs of Figure 5 for two galaxies in our sample. The over-density of stars along these lines are apparent by eye. The spread in color of the $\mathrm{BHeB}$ populations is due to photometric uncertainties, differential extinction, and the longer periods of time $\mathrm{BHeB}$ spend near the blue extrema in their evolution. Note that systems with significant amounts of differential extinction blend the MS stars with $\mathrm{BHeB}$ stars. In contrast, the galaxies in our sample all show this over-density of $\mathrm{BHeB}$ clearly separable from the MS in their CMDs, making selection of the $\mathrm{BHeB}$ stars viable.

Our second approach to isolating $\mathrm{BHeB}$ and $\mathrm{RHeB}$ stars follows the same methodology used to isolate the 

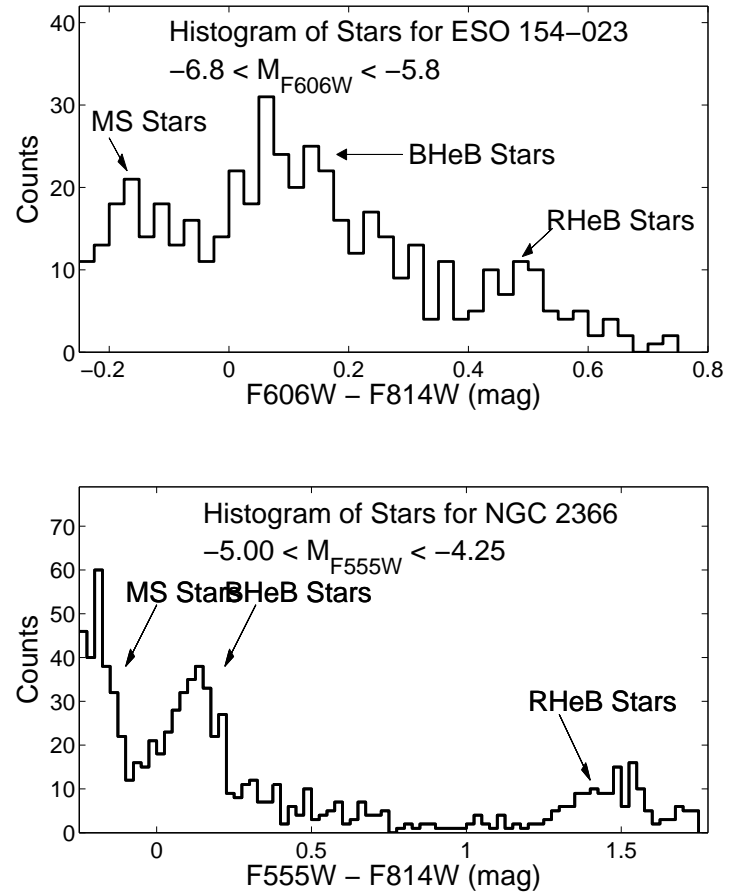

FIG. 6.- Example histograms of the stars in ESO154-023 (top) and NGC 2366 (bottom) in a magnitude slice of the CMDs shown in Figure 5 to be compared with theoretical histograms in Figure 3 The three peaks in the histograms correspond to the $\mathrm{MS}, \mathrm{BHeB}$ and $\mathrm{RHeB}$ populations. The color separation between the difference sequences is evident in the figure allowing the luminosity and color of the HeB sequences to be unambiguously identified. We chose the peak of the blue and red HeB histogram peaks to define the sequences.

$\mathrm{BHeB}$ in the synthetic CMDs (see $\$ 2$ ). We generate histograms of color for all stars in the $\mathrm{MS}, \mathrm{BHeB}$, and $\mathrm{RHeB}$ regions in magnitude intervals varying from $0.5 \mathrm{mag}$ to $1.0 \mathrm{mag}$, depending on the photometric uncertainties and SFH of each galaxy. Six magnitude intervals were used for the data instead of the nine used for the synthetic CMDs, as the location of the observed $\mathrm{HeB}$ sequences are measurable over a smaller range in luminosity than the synthetic HeB sequences. We present two example histograms in Figure 6 for data of shallower photometric depth (top panel; ESO 154-023) and for data of deeper photometric depth (bottom panel; NGC 2366). The histograms show the number of stars at each $V-I$ color in a sample magnitude interval. In each panel, there are three peaks corresponding to the MS, $\mathrm{BHeB}$, and $\mathrm{RHeB}$ stars.

We compared the colors and luminosities of the $\mathrm{BHeB}$ and the RHeB sequences that were defined by our two different methods. The sequences identified from the color histogram are plotted as solid lines in Figure 5, and the sequences identified by eye are drawn as dotted lines. The sequences are in good agreement with each other. For the remainder of the paper, we adopt the sequences selected with the aid of stellar histograms and interpolated using a quadratic function to generate our empirical sequences.

The measurement of the $\mathrm{HeB}$ sequences can be made down to a lower luminosity limit where the sequences begin to blend with other features in an optical CMD. This lower luminosity limit depends on both the underlying structure in the CMD and the photometric errors.
Specifically, the BHeB stars begin to merge with the red clump stars at fainter magnitudes while the $\mathrm{RHeB}$ stars blend with RGB stars. For example, for ESO 154-023 and NGC 2366 (Figure 5) the $\mathrm{BHeB}$ stars begin to merge into the red clump at $\sim \mathrm{M}_{V} \sim-3, V-I \sim 0.3$, and $\mathrm{M}_{V} \sim-2, V-I \sim 0.4$, respectively. Similarly, the $\mathrm{RHeB}$ begin to merge with the RGB stars at $\mathrm{M}_{V} \sim-4$, $V-I \sim 0.8$ and $\mathrm{M}_{V} \sim-3.8, V-I \sim 1$ respectively in the same galaxies. Since the position of the RGB stars, $\mathrm{HeB}$ stars, and the red clump depends on the metallicity and the SFH (Cole 1998), the lowest luminosity at which one can select HeBs from a CMD must be determined on a case-by-case basis.

We use synthetic stellar populations to determine the luminosity and color for unambiguously separating $\mathrm{HeB}$ stars from other stars in each galaxy. We generated synthetic CMDs using the SFHs derived in McQuinn et al. (2010b, and references therein), assumed a Salpeter IMF, and applied artificial star tests to mimic the uncertainties and completeness in the observed CMDs. Thus, the synthetic CMDs are close replicas of the observed CMDs, with the advantage that each star has a known age. We selected by eye the stars in the $\mathrm{BHeB}$ region, extending to depths expected to contain red clump stars, and stars in the $\mathrm{RHeB}$ region, extending to depths expected to contain RGB stars. The age distribution of these stars as a function of magnitude reveals the magnitude at which the young $\mathrm{HeB}$ stars become mixed with other stars. We define the lower luminosity limit for selecting $\mathrm{HeB}$ stars in each galaxy by the luminosity at which the young $\mathrm{HeB}$ stars become contaminated by $\sim 5 \%$ of MS or RGB stars.

For illustrative purposes, in Figure 7 we present examples of the age distribution as a function of magnitude of candidate $\mathrm{HeB}$ stars for a lower metallicity galaxy (left panels: $Z \sim 0.002$, UGC 9128) and for a higher metallicity galaxy (right panels: $Z \sim 0.008$, NGC 4449). The synthetic CMDs generated for this demonstration have SFRs that increase at recent times to ensure all time bins are well-populated. From Figure 7, there is a one-to-one correspondence of age with magnitude at brighter luminosities, but once the HeB populations become mixed with older stars, the age distribution spreads dramatically. From the top panels in Figure 7 the BHeB stars blend with the older red clump populations at $\simeq 400$ Myr and $\simeq 300 \mathrm{Myr}$ for the lower and higher metallicity systems respectively. Note that the brighter luminosities of lower metallicity stars means the $\mathrm{HeB}$ stars can be traced farther back in time at lower metallicities. At fainter magnitudes, the photometric uncertainties are higher, thus the $\mathrm{BHeB}$ region analyzed also contains some younger MS stars, as indicated in the figure. From the bottom panels of Figure 7, the RHeB stars blend with the older RGB population at $\lesssim 300 \mathrm{Myr}$ and $\lesssim 175 \mathrm{Myr}$ for the lower and higher metallicity systems. Comparing the age limits of the blue and red $\mathrm{HeB}$ stars for these two galaxies, Figure 7 illustrates the more limited time interval probed when using $\mathrm{RHeB}$ stars as tracers of a galaxy's SFH compared with BHeB stars. At the faintest magnitudes analyzed, a few stars transitioning from the MS to RHeB stars are identified.

Note that the structure of the CMD changes at solar metallicities. At this metallicity, the RHeB stars are wellseparated from the RGB stars at lower luminosities than in low metallicity systems, but begin to merge with $\mathrm{BHeB}$ 

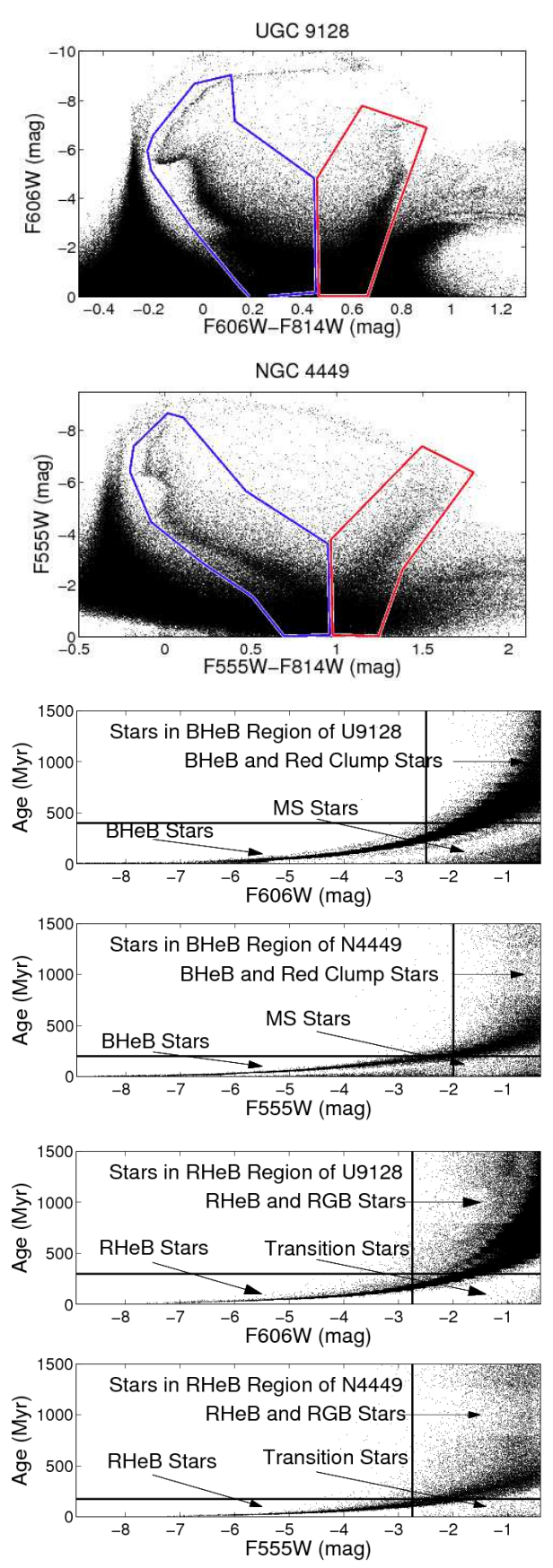

FIG. 7.- Examples of the age distribution of stars in the blue and red $\mathrm{HeB}$ regions of an optical CMD. In the top two panels, the synthetic CMD of a lower and higher metallicity galaxy are shown (UGC 9128, $Z \sim 0.002$ and NGC 4449, $Z \sim 0.009$ ). For illustrative purposes, we increased the SFRs at recent times to generate this CMD to ensure all luminosities are well-populated in the CMD. Outlined are the regions where the blue and red HeB stars are lie. Note there are more stars in the UGC 9128 simulated data as the SFRs used to simulate this galaxy were higher. In the middle two panels, the age distribution as a function of luminosity is shown for the stars selected in the $\mathrm{BHeB}$ region of each CMD. The bottom two panels show the distribution for the stars selected in the RHeB region. The vertical and horizontal lines in the middle and lower panels mark the limits in magnitudes and ages that the HeB stars can be uniquely separated in these CMDs with a contamination of order $5 \%$ from non-HeB stars. Comparing the two systems, the $\mathrm{BHeB}$ can be separated for longer times at lower metallicity and for longer times than the RHeB stars at all metallicities. The $\mathrm{RHeB}$ stars becomes difficult to separate at younger ages in higher metallicity systems reducing their usefulness in tracing the recent SFHs in these systems.

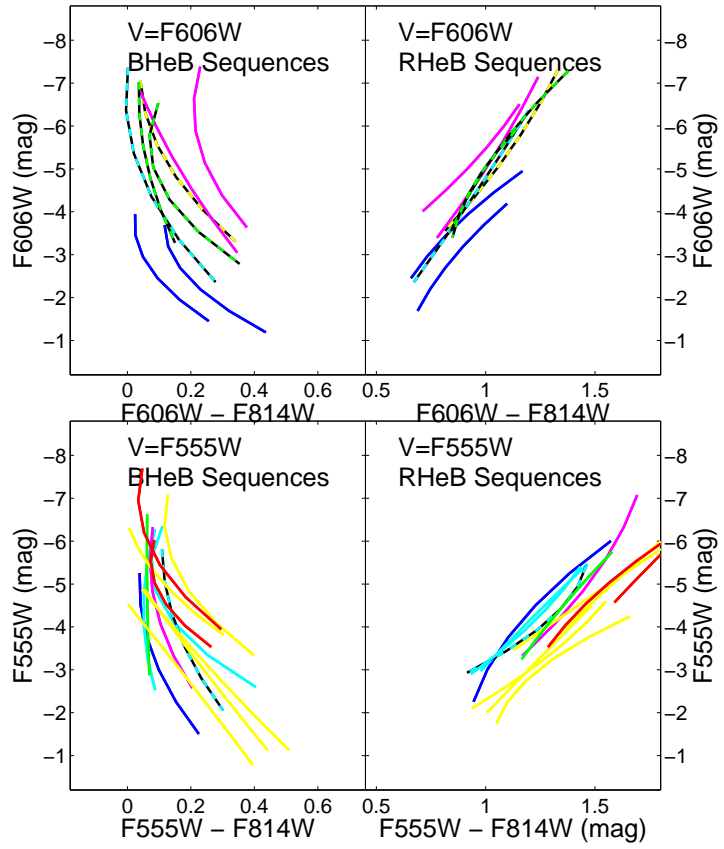

Fig. 8.- Empirical $\mathrm{BHeB}$ and $\mathrm{RHeB}$ sequences for two filter combinations. The colors represent the different metallicities of the galaxies accorinding to the following key: blue is $Z=0.001$, cyan is $Z=0.002$, green is $Z=0.003$, yellow is $Z=0.005$, magenta is $Z=0.006$, red is $Z=0.009$. The colored lines were dashed with black for galaxies lacking $\mathrm{H}$ II region abundances and whose metallicity was estimated from the SFHs. These sequences can be directly compared with the theoretically predicted sequences shown in Figure 4

stars after $~ 100 \mathrm{Myr}$, as the "blue loop" is shortened to $\lesssim 0.2 \mathrm{mag}$ in color. The merged HeB populations have a range in age of $\lesssim 100 \mathrm{Myr}$, thus the combined blue and red $\mathrm{HeB}$ stars can be used jointly to trace SF over timescales of order a few 100 Myr.

\subsection{The Empirically-Determined BHeB and $\mathrm{RHeB}$ Sequences}

The empirically determined red and blue $\mathrm{HeB}$ sequences are plotted in Figure 8 for the galaxy sample. In the top and bottom panels, we present the sequences for the galaxies observed with the F555W and F814W filters and the F606W and F814W filters respectively. The sequences are color-coded by present-day metallicity values, with blue being the most metal poor $(0.0005<Z<0.0015)$, red being the most metal rich $(0.0080<Z<0.0095)$, and the other colors spanning the intermediate metallicities. The colors of the lines match those used for the theoretical sequences in Figure 4. For metallicities derived from $\mathrm{H}$ II region abundances, the lines in Figure 8 are drawn as solid colors; for metallicities derived from the luminosity-metallicity relation, the lines are dashed with black.

\section{COMPARING THE EMPIRICAL AND THEORETICAL HEB RESULTS}

The empirically determined blue and red $\mathrm{HeB}$ sequences in Figure 8 provide a general assessment of the location of the HeB stars for a given metallicity and filter combination and can be directly compared with the theoretically predicted $\mathrm{HeB}$ sequences in Figure 4. For easier comparison, we selected the sequences from one filter combination, F555W and F814W, and plot both 


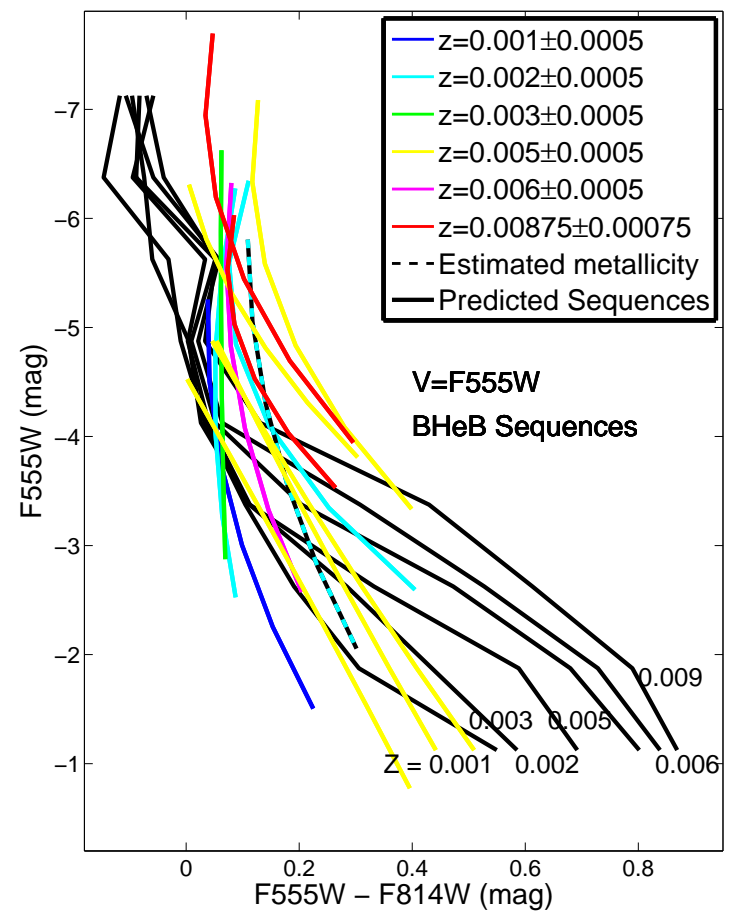

FIG. 9. - The empirical BHeB sequences measured for the F555W and $\mathrm{F} 814 \mathrm{~W}$ filter observations from Figure 8 plotted over the predicted sequences from Figure 4 The colors represents the metallicity of the galaxies sample as detailed in the figure legend. The predicted sequences are drawn in gray but span the same metallicity as the data. There is general agreement between the measured and predicted $\mathrm{BHeB}$ sequences. Differences exist with the theory predicted bluer (i.e., hotter) colors at brighter magnitudes than measured; the redder colors measured is likely due to extinction still present around these youngest BHeB stars.

the empirical and theoretical $\mathrm{BHeB}$ sequences together in Figure 9. Comparable RHeB sequences are plotted in Figure 10. We discuss each sequence separately followed by a comparison of the ratios of the number of stars in each sequence.

\subsection{Comparing BHeB Sequences}

Figure 9 shows that the empirically determined and theoretically predicted $\mathrm{BHeB}$ optical luminosities and colors agree in two important characteristics. First, both sets of HeB sequences overlap in the CMDs and follow comparable trends between color and luminosities along an individual sequence (i.e., bluer colors at higher luminosities and redder colors at lower luminosities). Second, higher metallicity stars follow redder sequences in both the observed and synthetic CMDs.

There are also two main differences between the measured and predicted $\mathrm{BHeB}$ sequences. First, at lower luminosities $\left(\mathrm{M}_{V} \gtrsim-4 \mathrm{mag}\right)$ the slope of the observed sequences (i.e., $\Delta \mathrm{V} / \Delta(\mathrm{V}-\mathrm{I})$ ) is steeper than the slope of the predicted sequences, indicating that the lower mass $\mathrm{BHeB}$ are observed to be bluer than predicted. Although the photometric uncertainties are largest at these fainter magnitudes, they are uniformly distributed in color and would not bias the measurement of the empirical sequences towards bluer colors. This difference cannot be explained by photometric uncertainties or by extinction since the empirical sequences would be redder in color than observed.

Second, we note an offset in color between the empiri- cal and theoretical sequences at intermediate and higher luminosities $\left(\mathrm{M}_{V} \lesssim-4 \mathrm{mag}\right)$. While both sets of sequences converge to a narrow range in color at high luminosities, the predicted sequences converge around F606W-F814W $\sim-0.10$ while the empirical sequences converge to redder colors of F606W-F814W $\sim 0.05$, an offset in color of $\sim 0.15 \mathrm{mag}$. At these higher luminosities, the $\mathrm{BHeB}$ population is made up of very young $(\mathrm{t} \lesssim 50$ Myr $)$, massive $\left(\mathrm{M} \gtrsim 7 \mathrm{M}_{\odot}\right)$ stars. The redder colors of the empirically derived sequences may be attributable to dust co-located with these massive stars. To test this possibility, we added varying amounts of extinction to a set of synthetic CMDs and re-measured the location of the predicted $\mathrm{BHeB}$ sequences. The addition of $\mathrm{A}_{V} \sim 0.5 \mathrm{mag}$ of extinction applied to the young stellar population brighter than $\mathrm{M}_{V} \sim 4$ mag shifts the predicted sequences to redder colors, closely matching the empirical sequences. This amount of extinction is consistent with the estimated reddening found around OB stars in the Large Magellenic Cloud (Harris et al. 1997). Thus, while we cannot rule out the possibility that the predicted colors reflect a discrepancy in the models, the observed redder colors of the brightest $\mathrm{BHeB}$ stars may be attributable to extinction. However, the addition of extinction would make the discrepancy worse for lower mass $\mathrm{BHeB}$ stars $\left(\lesssim 7 \mathrm{M}_{\odot}\right)$, if the dusty environment persists for longer timescales $(50-100 \mathrm{Myr})$.

\subsection{Comparing RHeB Sequences}

Like the BHeB sequences, the observed and theoretical RHeB sequences are in general agreement (Figure [10). The empirical and theoretical RHeB sequences both show a trend of higher metallicity sequences having redder colors.

However, there are greater differences between the measured and predicted $\mathrm{RHeB}$ sequences than the BHeB sequences (see \$4.1). First, the empirical RHeB sequences are redder, by as much as $\sim 0.5 \mathrm{mag}$, than the theoretical sequences at higher luminosities $\left(\mathrm{M}_{V} \lesssim-4\right.$ $\mathrm{mag})$, for an equivalent metallicity. Unlike the $0.15 \mathrm{mag}$ color offset in the BHeB sequences at bright magnitudes, this color difference cannot be fully attributable to differential extinction associated with young massive stars. Over three times the amount of extinction needed to explain the discrepancy in the BHeB sequences would be needed to account for the color shift in the RHeB sequences in spite of the stars being comparable masses and ages. Thus, unlike the difference between the observed and predicted color of the brightest BHeB stars, the bluer predicted colors of the brightest RHeB stars cannot be solely due to extinction.

Second, as seen in Figure 10, the slopes of the empirical sequences fall in a tight range $(\Delta \mathrm{F} 555 \mathrm{~W} / \Delta(\mathrm{F} 555 \mathrm{~W}$ $\mathrm{F} 814 \mathrm{~W})$ is between -3.9 and -7.0 with an average value of -4.9$)$. In contrast, the slopes of the theoretical sequences are a factor of $\sim 3$ steeper ranging from -13 to -25 with an average of -18 . The shallower slope of the empirical RHeB populations is thus a notable challenge for stellar models.

\subsection{Comparing the Empirical and Theoretical $B / R$} Ratios

We can further test stellar evolution models by measuring the ratio of the blue to red HeB stars. For the 


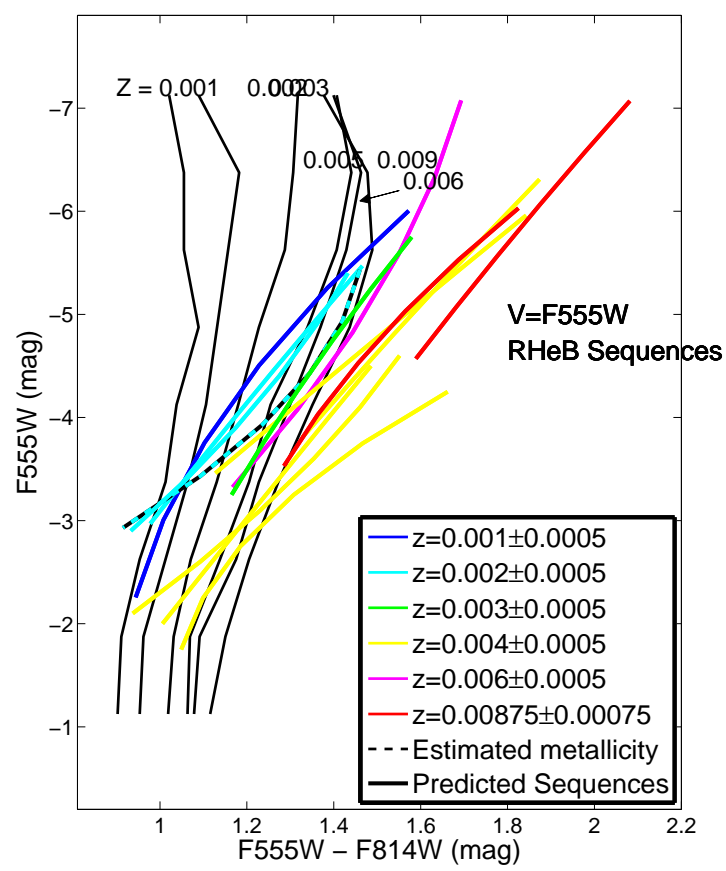

FIG. 10.- The empirical RHeB sequences measured for the F555W and F814W filter observations from Figure 8 plotted over the predicted sequences from Figure 4 The colors represents the metallicity of the galaxies sample as detailed in the figure legend. The predicted sequences are drawn in black and span the same metallicity as the data. There is general agreement between the measured and predicted $\mathrm{BHeB}$ sequences. Differences exist with the theory predicted bluer colors at brighter magnitudes than measured; however, unlike the $\mathrm{BHeB}$ stars, this color difference is too large to be mostly attributable to extinction around this very young population. In addition, the overall slope of the predicted sequences (i.e., $\Delta \mathrm{F} 555 \mathrm{~W} / \Delta(\mathrm{F} 555 \mathrm{~W}-\mathrm{F} 814 \mathrm{~W}))$ is steeper than measured by a factor of 3 .

synthetic CMDs, we select HeB stars within a width of $\pm 0.1 \mathrm{mag}$ in color around each sequence (see Figure 2). The small range in color is sufficient as the synthetic CMDs have no photometric uncertainty or extinction to widen the sequences in color. For the observed CMDs, the widths of the bins were selected on a case-by-case basis to best account for changing photometric uncertainties and extinction conditions (see Figure 5 as an example of the photometric uncertainties as a function of magnitude).

We measured the number of blue and red HeB stars as a function of magnitude. Using the stellar evolution isochrones, the magnitudes of both $\mathrm{HeB}$ phases were correlated with age. We matched the $\mathrm{BHeB}$ and $\mathrm{RHeB}$ stars of equal ages to calculate the ratio of $\mathrm{B} / \mathrm{R}$ stars. In Table 3. we list the number of blue and red HeB stars as a function of luminosity with the corresponding age determined from theoretical isochrones at the appropriate metallicity. In Figure 11, we present the average B/R ratios measured per metallicity bin for the observed (solid lines) and theoretical CMDs (dashed lines). Although the plots extend to $250 \mathrm{Myr}$, we limit the observed ratio to ages where contamination from other evolutionary phases is less than $5 \%$ as described in 3.3 . The $\mathrm{B} / \mathrm{R}$ ratios can be measured over the longest time periods at low metallicity, for which RHeB stars have brighter luminosities and are thus isolated in CMD space longer than higher metallicity RHeB stars. The exception to this is the $Z=0.005$ bin, which includes data from three fields in NGC 6822. These data are not only deeper due to the galaxy's proximity (such that the photometric uncertainties remain smaller at fainter magnitudes), but the $\mathrm{SFH}$ of NGC 6822 is such that the RHeB stars are distinguishable for longer times. In the highest metallicity bin of our sample $\left(Z=0.0087 \pm 0.00075 \simeq 0.5 \mathrm{Z}_{\odot}\right)$, the $\mathrm{RHeB}$ can only be robustly separated for $\lesssim 100$ Myr, providing a limited comparison with the predicted $\mathrm{B} / \mathrm{R}$ ratio.

The uncertainties on the empirical $\mathrm{B} / \mathrm{R}$ ratios in Figure 11 include both Poisson errors and photometric uncertainties. The photometric uncertainties were calculated by measuring the difference between the $\mathrm{B} / \mathrm{R}$ ratios from synthetic CMDs generated using artificial stars and from synthetic CMDs generated without applying artificial stars. At longer look-back times the uncertainty is dominated by the photometric uncertainties, because the larger number of fainter stars reduces the Poisson error. Note that in cases where the SFRs are rapidly changing, the $\mathrm{B} / \mathrm{R}$ ratio is dependent on the $\mathrm{SFH}$ of a galaxy. However, given the stability of these ratios with time, any effect of the $\mathrm{SFH}$ is within our range of uncertainties. We tested the effects photometric crowding has on the $\mathrm{B} / \mathrm{R}$ ratios by applying different crowding cuts to the photometry as stringent as 0.1 (see Dolphin 2000, and the DOLPHOT photometry manual for a explanation of photometric crowding measurements). The $\mathrm{B} / \mathrm{R}$ ratios were consistent across all crowding cuts, as the blue and red $\mathrm{HeB}$ stars are affected approximately equally by crowding.

We find that the empirical and predicted $B / R$ ratios are consistent, within the uncertainties, for nearly all time bins older than $50 \mathrm{Myr}$. Two sample KolmogorovSmirnov tests found the predicted and observed distribution of $B / R$ ratios satisfied the null hypothesis of the observed and predicted ratios being drawn from the same distribution for each metallicity bin. For the youngest $\mathrm{HeB}$ stars studied $(20 \mathrm{Myr}<$ Age $<50 \mathrm{Myr}$ ), the predicted ratios are higher than we observe in all metallicities bins. A similar result was reported by Dohm-Palmer \& Skillman (2002) for Sextans A with a metallicity of $Z=0.001$. These authors find the theoretical isochrones over-predicted the $\mathrm{B} / \mathrm{R}$ ratio by a factor of $\simeq 2$.

\section{DISCUSSION}

Our result that the predicted $\mathrm{HeB}$ sequences and $\mathrm{B} / \mathrm{R}$ ratios agree to first order with the observed sequences and $\mathrm{B} / \mathrm{R}$ ratios confirms the usefulness of applying these isochrones to interpret observations of stellar populations. The discrepancies we report are likely to have subtle, second order effects on these interpretations. The reconstruction of SFHs leverages information from all stellar populations in a CMD, so that isolating the effects of model inadequacies in the $\mathrm{HeB}$ stars alone is complex. While measuring the impact of these discrepancies on SFH recovery is outside the scope of this work, we can make some initial speculations 5 . We discuss the potential effects of the four discrepancies in turn.

First, the discrepancies found between the observed and predicted $\mathrm{BHeB}$ sequences for $\mathrm{M}_{V} \gtrsim-4$ mag are

\footnotetext{
${ }^{5}$ We refer the reader to references listed in $₫ 1$ for descriptions of deriving SFHs and McQuinn et al. (2010a) for an example of this application.
} 


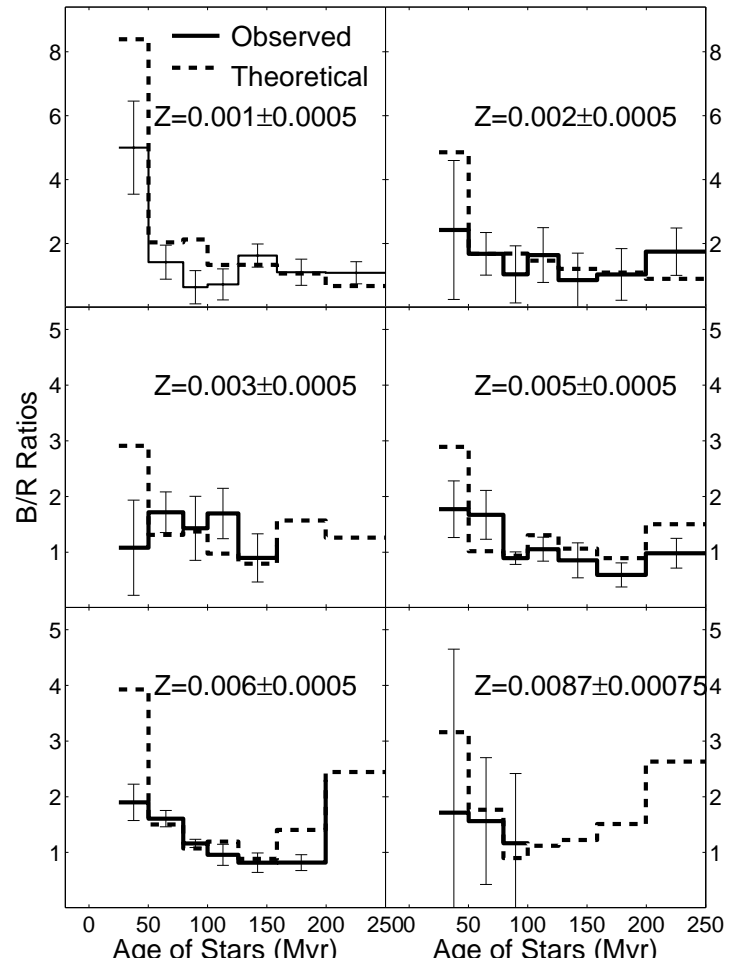

FIG. 11. - Plotted are the average $B / R$ ratios per metallicity bin for all galaxies in the sample (solid lines) and the $B / R$ ratios per metallicity bin predicted from theoretical isochrones (dotted lines). The predicted $\mathrm{B} / \mathrm{R}$ ratios are in general agreement with the measured $\mathrm{B} / \mathrm{R}$ ratios. The most notable difference is the over-prediction of the $B / R$ ratios for the youngest stars in the lowest metallicity studied confirming the results found by (Dohm-Palmer \& Skillman 2002) for Sextans A, a galaxy of similar metallicity. The RHeB stars become difficult to isolate unambiguously from RGB stars at higher metallicities limiting the age over which the $\mathrm{B} / \mathrm{R}$ ratio can be calculated. In Table 3 we list the number of blue and red $\mathrm{HeB}$ stars counted in each luminosity bins and the corresponding isochronal age.

likely due to factors external to the stellar evolution models and thus must be accounted for separately when applying isochrones. The youngest $\mathrm{HeB}$ stars are often still co-located with material from their nascent gas cloud whose extinction likely accounts for the redder than predicted colors observed for these stars. Derivations of the SFHs include additional $A_{V}=0.5 \mathrm{mag}$ of differential extinction to the models for stars younger than $40 \mathrm{Myr}$ with a linear ramp-down of extinction with age for stars between 40-100 Myr (Dolphin 2002), thus fitting the observed colors of the youngest HeB stars more closely. Second, the bluer color of the lower luminosity $\mathrm{BHeB}$ stars is likely to have the largest impact of the discrepancies on the derived SFRs. Stars in this region of the CMD may be mis-identified by the models as MS stars which can have a range in age at a given luminosity instead of the one-to-one age-luminosity relation of $\mathrm{HeB}$ stars. The color differences in the $\mathrm{RHeB}$ stars are greater for all magnitudes studied (i.e., the slopes of the observed and predicted $\mathrm{HeB}$ sequences as measured by $\Delta \mathrm{V} / \Delta(\mathrm{V}-\mathrm{I})$ do not match) which cannot be entirely due to extinction. We expect the effect of this color difference to be of small consequence in the derivation of the SFHs as the RHeB stars are well-isolated in an optical CMD above the RGB stars making confusion of this population with a different aged population unlikely. The mis-match in the slope of the RHeB sequences degrades the quality of the fit between the modeled and the observed stellar populations, but will likely have a negligible effect on the derived SFRs. Fourth, as the predicted B/R ratio for stars younger than $\sim 50 \mathrm{Myr}$ is too high, the derived SFRs may be underestimated, although this effect is mitigated as the derived SFRs also depend on the generally well-populated MS population.

A number of parameters in the stellar evolution models could explain the discrepancies between the predicted and observed red and blue $\mathrm{HeB}$ sequences and the $\mathrm{B} / \mathrm{R}$ ratios including the amount of internal mixing assumed (e.g., the effects of convective overshoot), ${ }^{12} \mathrm{C}(\alpha, \gamma){ }^{16} \mathrm{O}$ reaction rates, and the effects of stellar rotation and mass loss. Additionally, the stellar atmosphere models used to convert the surface luminosity and $T_{\text {eff }}$ to magnitudes and colors may affect the predicted luminosity and color of the HeB stars. While the stellar atmosphere models show good agreement to observed stellar populations (e.g., Girardi et al. 2002, 2008, and references therein), the atmosphere grids are incomplete for the brightest giants at lower metallicities and thus required extrapolation to reach brighter luminosities. This extrapolation could also explain our noted discrepancies, particularly the difference between the predicted and observed slopes of the RHeB sequences. Until the stellar evolution and atmospheric models are adjusted to describe the data with a higher degree of accuracy, it is premature to correct for these effects in the SFHs, particularly as the discrepancies are second order effects.

\section{CONCLUSIONS}

We find general agreement between the observed red and blue $\mathrm{HeB}$ sequences and the $\mathrm{B} / \mathrm{R}$ ratio in a sample of nineteen, starburst dwarf galaxies and the sequences predicted by Padova stellar evolution isochrones. We also find four notable differences between the empirical results and theoretical predictions.

- There is a difference in the color of HeB stars at brighter luminosities $\left(\mathrm{M}_{V} \lesssim-4 \mathrm{mag}\right)$, where the observed red and blue $\mathrm{HeB}$ sequences are redder than predicted by theoretical isochrones. We attribute at least part of the difference to the presence of $A_{V} \sim 0.5 \mathrm{mag}$ of extinction in the environs of the young HeB stars. However, while this extinction can produce the color difference between the predicted and observed $\mathrm{BHeB}$ sequences, it cannot account for the larger color difference between the predicted and observed $\mathrm{RHeB}$ sequences for $\mathrm{HeB}$ stars of comparable age. We note that for the bluest stars, U-band observations would provide the most leverage for comparing observed versus predicted effective temperatures.

- We see evidence that the observed BHeB stars are bluer than predicted by theoretical isochrones at lower luminosities $\left(\mathrm{M}_{V} \gtrsim-4 \mathrm{mag}\right)$. This difference cannot be explained by photometric uncertainties or additional extinction. Note that our conclusion is tentative as our sample is limited by photometric depth at these lower luminosities.

- The slope of the observed RHeB stars is shallower 
than predicted by the isochrones by more than a factor of 3 across the magnitude range studied.

- The theoretical isochrones over-predict the $\mathrm{B} / \mathrm{R}$ ratio for the most massive stars at all metallicities studied, confirming the findings reported in Dohm-Palmer \& Skillman (2002) for Sextans A $(Z=0.001)$.

Additionally, the measurement of the lower luminosity limit for unambiguously separating RHeB stars in optical CMDs shows that the $\mathrm{RHeB}$ stars are difficult to separate from RGB stars in the highest metallicity bin of our sample $\left(Z \sim 0.5 \mathrm{Z}_{\odot}\right)$ at ages as young as $100 \mathrm{Myr}$, reducing the effectiveness of using $\mathrm{RHeB}$ stars as tracers of a galaxy's SFH past $\sim 100 \mathrm{Myr}$ in higher metallicity systems. Further, we note the importance of studying a larger sample of galaxies containing substantial blue and red HeB populations, low extinction, spanning a range of metallicities. Future analysis is warranted comparing different stellar evolution models with these results.

\section{ACKNOWLEDGMENTS}

Support for this work was provided by NASA through a ROSES grant (No. NNX10AD57G). The authors are grateful to Philip Rosenfield for providing valuable comments on the stellar evolution models of HeB stars. E. D. S. is grateful for partial support from the University of Minnesota. K. B. W. M. gratefully acknowledges Matthew, Cole, and Carling for their support. We are grateful to the anonymous referee for her/his helpful comments and suggestions.

Facilities: Hubble Space Telescope

\section{REFERENCES}

Alongi, M., Bertelli, G., Bressan, A., Chiosi, C., Fagotto, F., Greggio, L., \& Nasi, E. 1993, A\&AS, 97, 851

Aparicio, A., Gallart, C., Chiosi, C., \& Bertelli, G. 1996, ApJ, 469, L97

Aparicio, A., \& Hidalgo, S. L. 2009, AJ, 138, 558

Asplund, M., Grevesse, N., Sauval, A. J., \& Scott, P. 2009, ARA\&A, 47, 481

Austin, S. M., Trentelman, G. F., \& Kashy, E. 1971, ApJ, 163, L79

Bertelli, G., Bressan, A. G., \& Chiosi, C. 1985, A\&A, 150, 33

Bertelli, G., Bressan, A., Chiosi, C., Fagotto, F., \& Nasi, E. 1994, A\&AS, 106, 275

Boquien, M., et al. 2009, ApJ, 706, 553

Bressan, A., Fagotto, F., Bertelli, G., \& Chiosi, C. 1993, A\&AS, 100, 647

Bressan, A., Granato, G. L., \& Silva, L. 1998, A\&A, 332, 135

Bruzual, G., \& Charlot, S. 2003, MNRAS, 344, 1000

Cardelli, J. A., Clayton, G. C., \& Mathis, J. S. 1989, ApJ, 345, 245

Calzetti, D., Kinney, A. L., \& Storchi-Bergmann, T. 1994, ApJ, 429,582

Castelli, F. , \& Kurucz, R. L. 2003, in IAU Symp. 210, Modelling of Stellar Atmospheres, eds.N. Piskunov, W. W. Weiss , \& D. F. Gray (San Francisco: ASP), p. A20

Chiosi, C., Bertelli, G., \& Bressan, A. 1992, ARA\&A, 30, 235

Cole, A. A. 1998, ApJ, 500, L137

Croxall, K. V., van Zee, L., Lee, H., Skillman, E. D., Lee, J. C.,

Côté, S., Kennicutt, R. C., \& Miller, B. W. 2009, ApJ, 705, 723

Dohm-Palmer, R. C., et al. 1997, AJ, 114, 2527

Dohm-Palmer, R. C., \& Skillman, E. D. 2002, AJ, 123, 1433

Dolphin, A. E. 2000, PASP, 112, 1383

Dolphin, A. E., 2002, MNRAS, 332, 91

Fagotto, F., Bressan, A., Bertelli, G., \& Chiosi, C. 1994, A\&AS, 104, 365

Dotter, A., Chaboyer, B., Jevremović, D., Kostov, V., Baron, E., \& Ferguson, J. W. 2008, ApJS, 178, 89

Fagotto, F., Bressan, A., Bertelli, G., \& Chiosi, C. 1994, A\&AS, 105,39

Ford, H. C., et al. 1998, Proc. SPIE, 3356, 234

Gallart, C., Zoccali, M., \& Aparicio, A. 2005, ARA\&A, 43, 387

Girardi, L., Bressan, A., Bertelli, G., \& Chiosi, C. 2000, A\&AS, 141,371

Girardi, L., Bertelli, G., Bressan, A., Chiosi, C., Groenewegen, M. A. T., Marigo, P., Salasnich, B., \& Weiss, A. 2002, A\&A, 391,195

Girardi, L., Grebel, E. K., Odenkirchen, M., \& Chiosi, C. 2004, A\&A, 422, 205

Girardi, L., et al. 2008, PASP, 120, 583

Groenewegen, M. A. T. 2006, A\&A, 448, 181

Harris, J., Zaritsky, D., \& Thompson, I. 1997, AJ, 114, 1933

Harris, J., \& Zaritsky, D. 2001, ApJS, 136, 25

Hayashi, C., \& Cameron, R. C. 1962, ApJ, 136, 166

Hayashi, C., Hōshi, R., \& Sugimoto, D. 1962, Progress of Theoretical Physics Supplement, 22, 1
Hidalgo-Gámez, A. M., Masegosa, J., \& Olofsson, K. 2001, A\&A, 369, 797

Hidalgo-Gámez, A. M., Olofsson, K., \& Masegosa, J. 2001, A\&A, 367,388

Holtzman, J. A., et al. 1995, PASP, 107, 156

Iben, I., Jr. 1966, ApJ, 143, 516 \& Rey, S.-C. 2009, ApJ, 703, 614

Kobulnicky, H. A., \& Skillman, E. D. 1996, ApJ, 471, 211

Kobulnicky, H. A., Skillman, E. D., Roy, J.-R., Walsh, J. R., \&

Rosa, M. R. 1997, ApJ, 477, 679

Kobulnicky, H. A., \& Skillman, E. D. 1997, ApJ, 489, 636

Kurucz, R. L. 1992, in The Stellar Populations of Galaxies, ed. B.

Barbuy, A. Renzini (Dordrecht, Kluwer), IAU Symp., 149, 225

Langer, N., \& Maeder, A. 1995, A\&A, 295, 685

Ledoux, P. 1947, AJ, 52, 155

Lee, H., Skillman, E. D., \& Venn, K. A. 2006, ApJ, 642, 813

Leitherer, C., Ortiz Otálvaro, P. A., Bresolin, F., Kudritzki,

R.-P., Lo Faro, B., Pauldrach, A. W. A., Pettini, M., \& Rix,

S. A. 2010, ApJS, 189, 309

Lejeune, T., \& Schaerer, D. 2001, A\&A, 366, 538

Maeder, A., \& Meynet, G. 2001, A\&A, 373, 555

Marigo, P., Girardi, L., Bressan, A., Groenewegen, M. A. T., Silva, L., \& Granato, G. L. 2008, A\&A, 482, 883

Meynet, G., \& Maeder, A. 1997, A\&A, 321, 465

McCall, M. L. 2004, AJ, 128, 2144

McQuinn, K. B. W., Skillman, E. D., Cannon, J. M., Dalcanton, J. J., Dolphin, A., Stark, D., \& Weisz, D. 2009, ApJ, 695, 561

McQuinn, K. B. W., Skillman, E. D., Cannon, J. M., Dalcanton,

J. J., Dolphin, A., Hidalgo-Rodriguez, S., Holtzman, J., Stark, D. et al., 2010, ApJ, 721, 297

McQuinn, K. B. W., Skillman, E. D., Cannon, J. M., Dalcanton, J. J., Dolphin, A., Hidalgo-Rodriguez, S., Holtzman, J., Stark, D. et al., 2010, ApJ, in press

Piersimoni, A. M., Bono, G., Castellani, M., Marconi, G., Cassisi, S., Buonanno, R., \& Nonino, M. 1999, A\&A, 352, L63

Pietrinferni, A., Cassisi, S., Salaris, M., \& Castelli, F. 2004, ApJ, 612,168

Roy, J.-R., Belley, J., Dutil, Y., \& Martin, P. 1996, ApJ, 460, 284 Salpeter, E. E. 1955, ApJ, 121, 161

Sakashita, S., Ôno, Y., \& Hayashi, C. 1959, Progress of

Theoretical Physics, 21, 315

Schlegel, D. J., Finkbeiner, D. P., \& Davis, M. 1998, ApJ, 500, 525

Skillman, E. D., Kennicutt, R. C., \& Hodge, P. W. 1989, ApJ, 347,875

Skillman, E. D., Televich, R. J., Kennicutt, R. C., Jr., Garnett, D. R., \& Terlevich, E. 1994, ApJ, 431, 172

Skillman, E. D., Côté, S., \& Miller, B. W. 2003, AJ, 125, 610

Stothers, R. 1966, ApJ, 143, 91

Stothers, R., \& Chin, C.-W. 1968, ApJ, 152, 225

Stothers, R., \& Chin, C.-W. 1975, ApJ, 198, 407

Stothers, R., \& Chin, C.-W. 1976, ApJ, 204, 472

Stothers, R. B., \& Chin, C.-W. 1991, ApJ, 374, 288

Stothers, R. B., \& Chin, C.-W. 1992, ApJ, 390, 136

Tremonti, C. A., et al. 2004, ApJ, 613, 898 
Tolstoy, E., \& Saha, A. 1996, ApJ, 462, 672

VandenBerg, D. A., Swenson, F. J., Rogers, F. J., Iglesias, C. A., \& Alexander, D. R. 2000, ApJ, 532, 430

van Zee, L., Haynes, M. P., \& Salzer, J. J. 1997, AJ, 114, 2479
Yi, S., Demarque, P., Kim, Y.-C., Lee, Y.-W., Ree, C. H., Lejeune, T., \& Barnes, S. 2001, ApJS, 136, 417

Zaritsky, D., Kennicutt, R. C., Jr., \& Huchra, J. P. 1994, ApJ, 420,87 
TABLE 1

Galaxy Sample Extinction and Metallicity Values

\begin{tabular}{|c|c|c|c|c|c|c|c|c|}
\hline $\begin{array}{c}\text { Galaxy } \\
(1)\end{array}$ & $\begin{array}{l}\text { HST V-band } \\
\text { filter } \\
(2)\end{array}$ & $\begin{array}{c}A_{V}(\mathrm{mag}) \\
\text { McQuinn et al. (2010b) } \\
\text { (3) }\end{array}$ & $\begin{array}{c}A_{V}(\mathrm{mag}) \\
\text { Schlegel et al. (1998) } \\
\text { (4) }\end{array}$ & $\begin{array}{l}A_{814}(\mathrm{mag}) \\
\text { Inferred } \\
(5)\end{array}$ & $\begin{array}{c}A_{800}(\mathrm{mag}) \\
\text { Schlegel et al. (1998) } \\
\text { (6) }\end{array}$ & $\begin{array}{c}\text { Oxygen } \\
\text { Abundance } \\
(7)\end{array}$ & $\begin{array}{c}\text { Source } \\
(8)\end{array}$ & $\begin{array}{l}\text { Equivalent } \\
\text { Isochrone } \\
(9)\end{array}$ \\
\hline Antlia Dwarf & F606W & $0.20 \pm .04$ & 0.21 & 0.15 & 0.15 & 7.39 & 1 & 0.001 \\
\hline UGC 9128 & F606W & $0.20 \pm .04$ & 0.07 & 0.15 & 0.05 & 7.74 & 2 & 0.002 \\
\hline UGC 4483 & F555W & $0.10 \pm .04$ & 0.11 & 0.07 & 0.07 & 7.50 & 3 & 0.001 \\
\hline NGC 4163 & F606W & $0.00 \pm .04$ & 0.05 & 0.00 & 0.04 & 7.69 & 4 & 0.002 \\
\hline UGC 6456 & F555W & $0.05 \pm .04$ & 0.12 & 0.04 & 0.07 & 7.64 & 5 & 0.002 \\
\hline NGC 6789 & F555W & $0.15 \pm .04$ & 0.23 & 0.11 & 0.14 & 7.77 & 4 & 0.002 \\
\hline NGC 4068 & F606W & $0.00 \pm .03$ & 0.06 & 0.00 & 0.04 & 7.84 & 4 & 0.003 \\
\hline DDO 165 & F555W & $0.10 \pm .04$ & 0.08 & 0.07 & 0.05 & 7.80 & 6 & 0.002 \\
\hline IC 4662 & F606W & $0.50 \pm .04^{\mathrm{a}}$ & 0.19 & 0.36 & 0.14 & 8.17 & 7 & 0.006 \\
\hline ESO 154-023 & F606W & $0.10 \pm .04$ & 0.05 & 0.07 & 0.03 & 8.01 & 4 & 0.004 \\
\hline NGC 2366 & F555W & $0.20 \pm .04^{\mathrm{a}}$ & 0.12 & 0.15 & 0.07 & 8.19 & 8 & 0.006 \\
\hline NGC 625 & F555W & $0.00 \pm .04$ & 0.06 & 0.00 & 0.03 & 8.10 & 9 & 0.005 \\
\hline NGC 784 & F606W & $0.10 \pm .04$ & 0.16 & 0.07 & 0.12 & 8.05 & 4 & 0.004 \\
\hline Ho II & F555W & $0.10 \pm .04$ & 0.11 & 0.07 & 0.06 & 7.92 & 6 & 0.003 \\
\hline NGC 5253 & F555W & $0.20 \pm .04^{\mathrm{a}}$ & 0.19 & 0.15 & 0.11 & 8.10 & 10 & 0.005 \\
\hline NGC 6822 & F555W & $1.05 \pm 0.1^{\mathrm{a}}$ & 0.78 & 0.76 & 0.46 & 8.11 & 11 & 0.005 \\
\hline NGC 4214 & F555W & $0.15 \pm .04$ & 0.07 & 0.11 & 0.04 & 8.38 & 12 & 0.009 \\
\hline NGC 1569 & F606W & $1.80 \pm 0.1^{\mathrm{a}}$ & 1.87 & 1.31 & 1.36 & 8.19 & 13 & 0.006 \\
\hline NGC 4449 & F555W & $0.25 \pm .04^{\mathrm{a}}$ & 0.06 & 0.18 & 0.04 & 8.21 & 14 & 0.008 \\
\hline
\end{tabular}

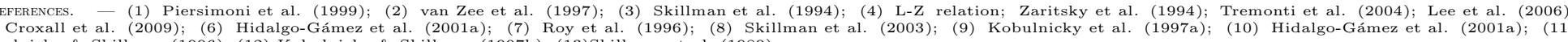

Note. - Col. (3) Estimated $\mathrm{A}_{V}$ includes both foreground and internal extinction whereas extinction estimates in Col. (4) from [Schlegel et al., (1998) measure only foreground extinction. Col McCall [2004). Col. (7) In 14 galaxies, oxygen abundances measurements were taken from the literature; in 5 galaxies oxygen abundances were estimated from the B-band luminosity-metallicity

a ${ }^{a}$ We report the highest $A_{V}$ of total extinction measured for these galaxies. Lower levels of extinction for measured for regions of lower surface brightness in these systems. 
TABLE 2

Instrument and Filter Combinations

\begin{tabular}{lcc}
\hline \hline HST Instrument & V band Filter & I band Filter \\
\hline ACS WFC & F606W & F814W \\
ACS WFC & F555W & F814W \\
WFPC2 & F555W & F814W
\end{tabular}

Note. - The observations were obtained using two HST imaging instruments and span three difference instrument-filter combinations. The theorectical isochrones for the ACS and WFPC2 instruments using the same filter bandpasses were indistinguishable from one another. Thus, we present only the results from each of the two unique filter combinations. Additionally, although our observations were not obtained with the bluer F475W filter, we include the predictions for the filter combination of $\mathrm{F} 475 \mathrm{~W}$ and $\mathrm{F} 814 \mathrm{~W}$ for comparison. 
Observational Constraints on Red and Blue Helium Burning Sequences

TABLE 3

Number of Blue and Red HeB stars as a Function of Age and LUMINOSITY

\begin{tabular}{|c|c|c|c|c|c|c|}
\hline $\begin{array}{l}\text { Log } \\
\text { Age }\end{array}$ & $\begin{array}{c}M_{V} \\
(\mathrm{mag})\end{array}$ & $\begin{array}{c}M_{F 814 W} \\
(\mathrm{mag})\end{array}$ & $\begin{array}{l}\text { No. BHeB } \\
\text { Stars }\end{array}$ & $\begin{array}{c}M_{V} \\
(\mathrm{mag})\end{array}$ & $\begin{array}{c}M_{F 814 W} \\
\text { (mag) }\end{array}$ & $\begin{array}{l}\text { No. RHeB } \\
\text { Stars }\end{array}$ \\
\hline \multicolumn{7}{|c|}{$\mathrm{NGC} 784, \mathrm{~V}=\mathrm{F} 606 \mathrm{~W}$} \\
\hline $\begin{array}{l}7.4 \\
7.5 \\
7.6 \\
7.7 \\
7.8 \\
7.9 \\
8.0 \\
8.1 \\
8.2\end{array}$ & $\begin{array}{l}-6.20 \\
-6.13 \\
-5.71 \\
-5.13 \\
-4.61 \\
-4.29 \\
-3.89 \\
-3.51 \\
-3.17\end{array}$ & $\begin{array}{l}-6.18 \\
-6.18 \\
-5.77 \\
-5.12 \\
-4.60 \\
-4.29 \\
-3.91 \\
-3.58 \\
-3.33\end{array}$ & $\begin{array}{r}36 \\
4 \\
58 \\
168 \\
267 \\
269 \\
483 \\
785 \\
1176\end{array}$ & $\begin{array}{l}-6.24 \\
-5.89 \\
-5.48 \\
-5.10 \\
-4.69 \\
-4.24 \\
-3.86 \\
-3.41 \\
-2.99\end{array}$ & $\begin{array}{l}-7.27 \\
-6.91 \\
-6.47 \\
-6.05 \\
-5.63 \\
-5.15 \\
-4.73 \\
-4.26 \\
-3.82\end{array}$ & $\begin{array}{r}60 \\
42 \\
63 \\
123 \\
158 \\
257 \\
400 \\
914 \\
1795\end{array}$ \\
\hline \multicolumn{7}{|c|}{$\mathrm{IC} 4662, \mathrm{~V}=\mathrm{F} 606 \mathrm{~W}$} \\
\hline $\begin{array}{l}7.4 \\
7.5 \\
7.6 \\
7.7 \\
7.8 \\
7.9 \\
8.0 \\
8.1 \\
8.2 \\
8.3 \\
8.4\end{array}$ & $\begin{array}{l}-6.24 \\
-6.09 \\
-5.68 \\
-5.19 \\
-4.71 \\
-4.37 \\
-3.95 \\
-3.62 \\
-3.17 \\
-2.69 \\
-2.18\end{array}$ & $\begin{array}{l}-6.25 \\
-6.13 \\
-5.73 \\
-5.21 \\
-4.72 \\
-4.40 \\
-4.01 \\
-3.75 \\
-3.41 \\
-3.05 \\
-2.67\end{array}$ & $\begin{array}{r}35 \\
11 \\
33 \\
80 \\
124 \\
137 \\
259 \\
307 \\
605 \\
828 \\
\mathrm{NaN}\end{array}$ & $\begin{array}{l}-6.29 \\
-5.93 \\
-5.53 \\
-5.06 \\
-4.74 \\
-4.32 \\
-3.84 \\
-3.47 \\
-2.97 \\
-2.79 \\
-2.45\end{array}$ & $\begin{array}{l}-7.35 \\
-6.99 \\
-6.54 \\
-6.04 \\
-5.71 \\
-5.26 \\
-4.74 \\
-4.34 \\
-3.82 \\
-3.63 \\
-3.26\end{array}$ & $\begin{array}{r}56 \\
30 \\
62 \\
84 \\
76 \\
131 \\
260 \\
380 \\
799 \\
372 \\
636\end{array}$ \\
\hline $\begin{array}{l}\text { Antli } \\
7.4 \\
7.5 \\
7.6 \\
7.7 \\
7.8 \\
7.9 \\
8.0 \\
8.1 \\
8.2 \\
8.3 \\
8.4 \\
8.5 \\
8.6 \\
8.7\end{array}$ & $\begin{array}{l}\mathrm{V}=\mathrm{F} 6 \mathrm{C} \\
-5.65 \\
-5.54 \\
-5.28 \\
-4.99 \\
-4.60 \\
-4.32 \\
-3.93 \\
-3.65 \\
-3.36 \\
-2.93 \\
-2.55 \\
-2.21 \\
-1.78 \\
-1.33\end{array}$ & $\begin{array}{l}6 \mathrm{~W} \\
-5.58 \\
-5.51 \\
-5.26 \\
-4.98 \\
-4.60 \\
-4.33 \\
-3.96 \\
-3.71 \\
-3.46 \\
-3.07 \\
-2.70 \\
-2.33 \\
-1.99 \\
-1.66\end{array}$ & $\begin{array}{r}0 \\
0 \\
0 \\
0 \\
0 \\
1 \\
1 \\
2 \\
2 \\
10 \\
27 \\
23 \\
29 \\
72\end{array}$ & $\begin{array}{l}-6.50 \\
-6.10 \\
-5.62 \\
-5.11 \\
-4.66 \\
-4.18 \\
-3.75 \\
-3.24 \\
-2.88 \\
-2.51 \\
-2.17 \\
-1.83 \\
-1.53 \\
-1.33\end{array}$ & $\begin{array}{l}-7.29 \\
-6.90 \\
-6.43 \\
-5.94 \\
-5.46 \\
-4.95 \\
-4.50 \\
-3.98 \\
-3.60 \\
-3.23 \\
-2.87 \\
-2.53 \\
-2.22 \\
-2.03\end{array}$ & $\begin{array}{r}0 \\
0 \\
0 \\
0 \\
1 \\
0 \\
3 \\
6 \\
7 \\
11 \\
27 \\
53 \\
32 \\
12\end{array}$ \\
\hline $\begin{array}{l}\text { UGC } \\
7.4 \\
7.5 \\
7.6 \\
7.7 \\
7.8 \\
7.9 \\
8.0 \\
8.1 \\
8.2 \\
8.3 \\
8.4 \\
8.5 \\
8.6 \\
8.7\end{array}$ & $\begin{array}{l}9128, \mathrm{~V}= \\
-5.89 \\
-5.76 \\
-5.64 \\
-5.06 \\
-4.60 \\
-4.24 \\
-3.98 \\
-3.60 \\
-3.19 \\
-2.88 \\
-2.44 \\
-2.01 \\
-1.62 \\
-1.20\end{array}$ & $\begin{array}{r}\text { F606W } \\
-5.85 \\
-5.75 \\
-5.66 \\
-5.05 \\
-4.60 \\
-4.25 \\
-4.00 \\
-3.65 \\
-3.31 \\
-3.05 \\
-2.73 \\
-2.35 \\
-2.02 \\
-1.67\end{array}$ & $\begin{array}{r}0 \\
0 \\
1 \\
3 \\
11 \\
4 \\
23 \\
56 \\
152 \\
117 \\
160 \\
313 \\
385 \\
464\end{array}$ & $\begin{array}{l}-6.45 \\
-6.01 \\
-5.59 \\
-5.10 \\
-4.71 \\
-4.21 \\
-3.83 \\
-3.57 \\
-3.05 \\
-2.64 \\
-2.28 \\
-1.94 \\
-1.72 \\
-1.45\end{array}$ & $\begin{array}{l}-7.38 \\
-6.93 \\
-6.50 \\
-6.01 \\
-5.59 \\
-5.06 \\
-4.66 \\
-4.39 \\
-3.84 \\
-3.41 \\
-3.04 \\
-2.69 \\
-2.47 \\
-2.22\end{array}$ & $\begin{array}{r}0 \\
0 \\
0 \\
2 \\
13 \\
24 \\
21 \\
20 \\
85 \\
138 \\
99 \\
72 \\
35 \\
0\end{array}$ \\
\hline $\begin{array}{l}\text { NGC } \\
7.4 \\
7.5 \\
7.6 \\
7.7 \\
7.8 \\
7.9 \\
8.0 \\
8.1 \\
8.2 \\
8.3 \\
8.4 \\
8.5\end{array}$ & $\begin{array}{c}4163, \mathrm{~V}= \\
-5.94 \\
-5.77 \\
-5.57 \\
-5.08 \\
-4.64 \\
-4.33 \\
-3.91 \\
-3.60 \\
-3.18 \\
-2.81 \\
-2.43 \\
-2.04\end{array}$ & $\begin{array}{r}F 606 \mathrm{~W} \\
-5.88 \\
-5.75 \\
-5.58 \\
-5.06 \\
-4.62 \\
-4.33 \\
-3.91 \\
-3.63 \\
-3.26 \\
-2.97 \\
-2.68 \\
-2.33\end{array}$ & $\begin{array}{r}3 \\
2 \\
2 \\
14 \\
23 \\
18 \\
40 \\
53 \\
44 \\
120 \\
159 \\
355\end{array}$ & $\begin{array}{l}-6.40 \\
-6.03 \\
-5.60 \\
-5.06 \\
-4.70 \\
-4.28 \\
-3.89 \\
-3.43 \\
-2.99 \\
-2.58 \\
-2.26 \\
-1.99\end{array}$ & $\begin{array}{l}-7.30 \\
-6.91 \\
-6.48 \\
-5.93 \\
-5.55 \\
-5.11 \\
-4.69 \\
-4.21 \\
-3.76 \\
-3.33 \\
-3.00 \\
-2.71\end{array}$ & $\begin{array}{r}8 \\
7 \\
8 \\
10 \\
11 \\
33 \\
35 \\
75 \\
53 \\
142 \\
126 \\
224\end{array}$ \\
\hline $\begin{array}{l}\text { NGC } \\
7.4 \\
7.5\end{array}$ & $\begin{array}{c}4068, \mathrm{~V}= \\
-6.04 \\
-5.99\end{array}$ & $\begin{array}{r}F 606 \mathrm{~W} \\
-5.99 \\
-6.00\end{array}$ & $\begin{array}{r}33 \\
1\end{array}$ & $\begin{array}{l}-6.37 \\
-5.96\end{array}$ & $\begin{array}{l}-7.32 \\
-6.90\end{array}$ & $\begin{array}{l}29 \\
17\end{array}$ \\
\hline
\end{tabular}


McQuinn, Skillman et al.

TABLE 3 - Continued

\begin{tabular}{|c|c|c|c|c|c|c|}
\hline $\begin{array}{l}\text { Log } \\
\text { Age }\end{array}$ & $\begin{array}{c}M_{V} \\
(\mathrm{mag})\end{array}$ & $\begin{array}{c}M_{F 814 W} \\
(\mathrm{mag})\end{array}$ & $\begin{array}{c}\text { No. BHeB } \\
\text { Stars }\end{array}$ & $\begin{array}{c}M_{V} \\
(\mathrm{mag})\end{array}$ & $\begin{array}{c}M_{F 814 W} \\
\text { (mag) }\end{array}$ & $\begin{array}{l}\text { No. RHeB } \\
\text { Stars }\end{array}$ \\
\hline $\begin{array}{l}7.6 \\
7.7 \\
7.8 \\
7.9 \\
8.0 \\
8.1 \\
8.2 \\
8.3\end{array}$ & $\begin{array}{l}-5.75 \\
-5.13 \\
-4.61 \\
-4.21 \\
-3.86 \\
-3.56 \\
-3.14 \\
-2.79\end{array}$ & $\begin{array}{l}-5.78 \\
-5.11 \\
-4.60 \\
-4.21 \\
-3.86 \\
-3.60 \\
-3.26 \\
-2.99\end{array}$ & $\begin{array}{r}15 \\
68 \\
110 \\
195 \\
216 \\
274 \\
442 \\
499\end{array}$ & $\begin{array}{l}-5.51 \\
-5.16 \\
-4.66 \\
-4.23 \\
-3.96 \\
-3.47 \\
-3.08 \\
-2.65\end{array}$ & $\begin{array}{l}-6.44 \\
-6.06 \\
-5.54 \\
-5.09 \\
-4.82 \\
-4.29 \\
-3.89 \\
-3.43\end{array}$ & $\begin{array}{r}25 \\
36 \\
77 \\
136 \\
109 \\
361 \\
490 \\
529\end{array}$ \\
\hline $\begin{array}{l}\text { ESO } \\
7.4 \\
7.5 \\
7.6 \\
7.7 \\
7.8 \\
7.9 \\
8.0 \\
8.1 \\
8.2 \\
8.3\end{array}$ & $\begin{array}{l}54-023, \\
-6.29 \\
-6.10 \\
-5.79 \\
-5.16 \\
-4.65 \\
-4.29 \\
-3.86 \\
-3.53 \\
-3.10 \\
-2.77\end{array}$ & $\begin{array}{c}\mathrm{V}=\mathrm{F} 606 \mathrm{~W} \\
-6.28 \\
-6.13 \\
-5.84 \\
-5.15 \\
-4.64 \\
-4.28 \\
-3.86 \\
-3.56 \\
-3.19 \\
-2.98\end{array}$ & $\begin{array}{r}42 \\
18 \\
51 \\
174 \\
304 \\
317 \\
641 \\
631 \\
1089 \\
\text { NaN }\end{array}$ & $\begin{array}{l}-6.30 \\
-5.99 \\
-5.59 \\
-5.10 \\
-4.71 \\
-4.26 \\
-3.87 \\
-3.51 \\
-3.10 \\
-2.80\end{array}$ & $\begin{array}{l}-7.29 \\
-6.97 \\
-6.54 \\
-6.03 \\
-5.61 \\
-5.13 \\
-4.72 \\
-4.36 \\
-3.91 \\
-3.60\end{array}$ & $\begin{array}{r}69 \\
33 \\
58 \\
125 \\
148 \\
286 \\
402 \\
611 \\
1233 \\
1142\end{array}$ \\
\hline $\begin{array}{l}\text { NGC } \\
7.4 \\
7.5 \\
7.6 \\
7.7 \\
7.8 \\
7.9 \\
8.0\end{array}$ & $\begin{array}{l}1569, \mathrm{~V}= \\
-6.04 \\
-5.95 \\
-5.54 \\
-5.02 \\
-4.52 \\
-4.27 \\
-3.86\end{array}$ & $\begin{array}{r}F 606 \mathrm{~W} \\
-6.16 \\
-6.12 \\
-5.71 \\
-5.17 \\
-4.67 \\
-4.45 \\
-4.06\end{array}$ & $\begin{array}{r}660 \\
65 \\
384 \\
752 \\
1058 \\
604 \\
961\end{array}$ & $\begin{array}{l}-6.26 \\
-5.79 \\
-5.42 \\
-5.08 \\
-4.65 \\
-4.20 \\
-3.82\end{array}$ & $\begin{array}{l}-7.42 \\
-6.94 \\
-6.53 \\
-6.16 \\
-5.69 \\
-5.23 \\
-4.84\end{array}$ & $\begin{array}{r}819 \\
385 \\
369 \\
382 \\
741 \\
1189 \\
1323\end{array}$ \\
\hline $\begin{array}{l}\text { UGC } \\
7.4 \\
7.5 \\
7.6 \\
7.7 \\
7.8 \\
7.9 \\
8.0 \\
8.1 \\
8.2 \\
8.3 \\
8.4 \\
8.5 \\
8.6 \\
8.7\end{array}$ & $\begin{array}{c}448, \mathrm{~V}= \\
-5.64 \\
-5.54 \\
-5.33 \\
-4.96 \\
-4.60 \\
-4.26 \\
-3.95 \\
-3.61 \\
-3.20 \\
-2.86 \\
-2.43 \\
-2.06 \\
-1.59 \\
-1.16\end{array}$ & $\begin{array}{r}F 555 \\
-5.54 \\
-5.50 \\
-5.30 \\
-4.94 \\
-4.59 \\
-4.27 \\
-3.97 \\
-3.67 \\
-3.30 \\
-3.00 \\
-2.64 \\
-2.26 \\
-1.87 \\
-1.57\end{array}$ & $\begin{array}{r}5 \\
1 \\
3 \\
4 \\
10 \\
11 \\
14 \\
26 \\
53 \\
66 \\
102 \\
156 \\
239 \\
332\end{array}$ & $\begin{array}{l}-6.15 \\
-5.71 \\
-5.25 \\
-4.80 \\
-4.29 \\
-3.84 \\
-3.45 \\
-3.05 \\
-2.63 \\
-2.31 \\
-1.93 \\
-1.59 \\
-1.30 \\
-1.11\end{array}$ & $\begin{array}{l}-7.25 \\
-6.81 \\
-6.35 \\
-5.90 \\
-5.37 \\
-4.88 \\
-4.48 \\
-4.05 \\
-3.62 \\
-3.27 \\
-2.89 \\
-2.55 \\
-2.25 \\
-2.06\end{array}$ & $\begin{array}{r}1 \\
0 \\
1 \\
3 \\
2 \\
10 \\
19 \\
15 \\
43 \\
44 \\
53 \\
51 \\
23 \\
0\end{array}$ \\
\hline $\begin{array}{l}\text { UGC } \\
7.4 \\
7.5 \\
7.6 \\
7.7 \\
7.8 \\
7.9 \\
8.0 \\
8.1 \\
8.2 \\
8.3 \\
8.4\end{array}$ & $\begin{array}{l}6456, \mathrm{~V}= \\
-5.78 \\
-5.65 \\
-5.48 \\
-5.02 \\
-4.57 \\
-4.29 \\
-3.92 \\
-3.53 \\
-3.17 \\
-2.80 \\
-2.37\end{array}$ & $\begin{array}{r}F 555 \mathrm{~W} \\
-5.70 \\
-5.63 \\
-5.49 \\
-5.01 \\
-4.55 \\
-4.29 \\
-3.94 \\
-3.56 \\
-3.31 \\
-3.00 \\
-2.64\end{array}$ & $\begin{array}{r}42 \\
5 \\
9 \\
16 \\
27 \\
30 \\
47 \\
66 \\
105 \\
150 \\
153\end{array}$ & $\begin{array}{l}-6.13 \\
-5.65 \\
-5.23 \\
-4.72 \\
-4.27 \\
-3.78 \\
-3.45 \\
-3.05 \\
-2.60 \\
-2.29 \\
-1.92\end{array}$ & $\begin{array}{l}-7.31 \\
-6.83 \\
-6.42 \\
-5.91 \\
-5.44 \\
-4.88 \\
-4.53 \\
-4.12 \\
-3.65 \\
-3.33 \\
-2.96\end{array}$ & $\begin{array}{r}7 \\
6 \\
6 \\
12 \\
11 \\
19 \\
15 \\
62 \\
126 \\
125 \\
\text { NaN }\end{array}$ \\
\hline $\begin{array}{l}\text { NGC } \\
7.4 \\
7.5 \\
7.6 \\
7.7 \\
7.8 \\
7.9 \\
8.0 \\
8.1 \\
8.2 \\
8.3 \\
8.4 \\
8.5\end{array}$ & $\begin{array}{l}6789, \mathrm{~V}= \\
-5.90 \\
-5.81 \\
-5.54 \\
-5.05 \\
-4.60 \\
-4.23 \\
-3.89 \\
-3.55 \\
-3.13 \\
-2.71 \\
-2.35 \\
-1.86\end{array}$ & $\begin{array}{r}F 606 \mathrm{~W} \\
-5.85 \\
-5.80 \\
-5.56 \\
-5.06 \\
-4.60 \\
-4.23 \\
-3.90 \\
-3.58 \\
-3.18 \\
-2.92 \\
-2.71 \\
-2.28\end{array}$ & $\begin{array}{r}4 \\
0 \\
2 \\
13 \\
14 \\
15 \\
40 \\
36 \\
88 \\
116 \\
188 \\
261\end{array}$ & $\begin{array}{l}-6.02 \\
-5.64 \\
-5.25 \\
-4.75 \\
-4.30 \\
-3.87 \\
-3.42 \\
-3.05 \\
-2.65 \\
-2.28 \\
-1.98 \\
-1.64\end{array}$ & $\begin{array}{l}-7.52 \\
-7.08 \\
-6.65 \\
-6.10 \\
-5.56 \\
-5.07 \\
-4.59 \\
-4.15 \\
-3.71 \\
-3.33 \\
-3.04 \\
-2.70\end{array}$ & $\begin{array}{r}5 \\
4 \\
2 \\
14 \\
22 \\
26 \\
63 \\
87 \\
117 \\
41 \\
0 \\
0\end{array}$ \\
\hline $\begin{array}{l}\text { DDO } \\
7.4 \\
7.5\end{array}$ & $\begin{array}{c}165, V= \\
-5.93 \\
-5.85\end{array}$ & $\begin{array}{r}555 \mathrm{~W} \\
-5.91 \\
-5.87\end{array}$ & $\begin{array}{r}25 \\
7\end{array}$ & $\begin{array}{l}-6.01 \\
-5.62\end{array}$ & $\begin{array}{l}-7.35 \\
-6.94\end{array}$ & $\begin{array}{l}11 \\
12\end{array}$ \\
\hline
\end{tabular}


TABLE 3 - Continued

\begin{tabular}{|c|c|c|c|c|c|c|}
\hline $\begin{array}{l}\text { Log } \\
\text { Age }\end{array}$ & $\begin{array}{c}M_{V} \\
(\mathrm{mag})\end{array}$ & $\begin{array}{c}M_{F 814 W} \\
\text { (mag) }\end{array}$ & $\begin{array}{l}\text { No. BHeB } \\
\text { Stars }\end{array}$ & $\begin{array}{c}M_{V} \\
(\mathrm{mag})\end{array}$ & $\begin{array}{c}M_{F 814 W} \\
\text { (mag) }\end{array}$ & $\begin{array}{l}\text { No. RHeB } \\
\text { Stars }\end{array}$ \\
\hline $\begin{array}{l}7.6 \\
7.7 \\
7.8 \\
7.9 \\
8.0 \\
8.1 \\
8.2 \\
8.3 \\
8.4\end{array}$ & $\begin{array}{l}-5.62 \\
-5.02 \\
-4.57 \\
-4.23 \\
-3.84 \\
-3.47 \\
-3.08 \\
-2.72 \\
-2.23\end{array}$ & $\begin{array}{l}-5.67 \\
-5.04 \\
-4.58 \\
-4.24 \\
-3.85 \\
-3.48 \\
-3.14 \\
-2.84 \\
-2.40\end{array}$ & $\begin{array}{r}23 \\
181 \\
299 \\
447 \\
633 \\
548 \\
818 \\
1049 \\
2782\end{array}$ & $\begin{array}{l}-5.13 \\
-4.79 \\
-4.29 \\
-3.87 \\
-3.39 \\
-3.02 \\
-2.65 \\
-2.31 \\
-1.93\end{array}$ & $\begin{array}{l}-6.42 \\
-6.07 \\
-5.53 \\
-5.08 \\
-4.56 \\
-4.15 \\
-3.76 \\
-3.39 \\
-3.00\end{array}$ & $\begin{array}{r}43 \\
55 \\
190 \\
291 \\
521 \\
420 \\
416 \\
441 \\
\text { NaN }\end{array}$ \\
\hline $\begin{array}{l}\text { NGC } \\
7.4 \\
7.5 \\
7.6 \\
7.7 \\
7.8 \\
7.9 \\
8.0 \\
8.1 \\
8.2 \\
8.3\end{array}$ & $\begin{array}{c}2366, \mathrm{~V}= \\
-6.04 \\
-5.96 \\
-5.56 \\
-5.02 \\
-4.66 \\
-4.23 \\
-3.87 \\
-3.48 \\
-3.00 \\
-2.43\end{array}$ & $\begin{array}{r}F 55 \mathrm{~W} \\
-6.05 \\
-5.97 \\
-5.59 \\
-5.03 \\
-4.68 \\
-4.26 \\
-3.91 \\
-3.57 \\
-3.31 \\
-2.85\end{array}$ & $\begin{array}{r}64 \\
7 \\
54 \\
150 \\
162 \\
334 \\
462 \\
939 \\
1866 \\
4079\end{array}$ & $\begin{array}{l}-5.71 \\
-5.30 \\
-4.92 \\
-4.57 \\
-4.17 \\
-3.82 \\
-3.41 \\
-2.97 \\
-2.57 \\
-2.21\end{array}$ & $\begin{array}{l}-7.30 \\
-6.91 \\
-6.50 \\
-6.12 \\
-5.70 \\
-5.34 \\
-4.91 \\
-4.46 \\
-4.05 \\
-3.69\end{array}$ & $\begin{array}{r}15 \\
21 \\
43 \\
79 \\
112 \\
173 \\
402 \\
928 \\
1768 \\
1100\end{array}$ \\
\hline $\begin{array}{l}\text { NGC } \\
7.4 \\
7.5 \\
7.6 \\
7.7 \\
7.8 \\
7.9 \\
8.0 \\
8.1 \\
8.2\end{array}$ & $\begin{array}{l}625, \mathrm{~V}= \\
-6.13 \\
-5.99 \\
-5.68 \\
-5.05 \\
-4.52 \\
-4.24 \\
-3.89 \\
-3.48 \\
-3.02\end{array}$ & $\begin{array}{c}555 \mathrm{~W} \\
-6.12 \\
-6.02 \\
-5.72 \\
-5.03 \\
-4.48 \\
-4.20 \\
-3.88 \\
-3.52 \\
-3.14\end{array}$ & $\begin{array}{r}28 \\
5 \\
9 \\
43 \\
99 \\
90 \\
139 \\
219 \\
299\end{array}$ & $\begin{array}{l}-5.81 \\
-5.42 \\
-5.10 \\
-4.74 \\
-4.24 \\
-3.89 \\
-3.51 \\
-3.17 \\
-2.71\end{array}$ & $\begin{array}{l}-7.21 \\
-6.80 \\
-6.44 \\
-6.04 \\
-5.51 \\
-5.12 \\
-4.72 \\
-4.34 \\
-3.87\end{array}$ & $\begin{array}{r}8 \\
19 \\
17 \\
32 \\
84 \\
92 \\
187 \\
222 \\
\text { NaN }\end{array}$ \\
\hline $\begin{array}{l}\text { Ho II } \\
7.4 \\
7.5 \\
7.6 \\
7.7 \\
7.8 \\
7.9 \\
8.0 \\
8.1 \\
8.2 \\
8.3\end{array}$ & $\begin{array}{l}\mathrm{V}=\mathrm{F} 55 \\
-6.10 \\
-6.06 \\
-5.76 \\
-5.14 \\
-4.63 \\
-4.30 \\
-3.87 \\
-3.53 \\
-3.13 \\
-2.72\end{array}$ & $\begin{array}{l}\text { W } \\
-6.09 \\
-6.05 \\
-5.79 \\
-5.15 \\
-4.63 \\
-4.31 \\
-3.89 \\
-3.59 \\
-3.26 \\
-3.00\end{array}$ & $\begin{array}{r}99 \\
12 \\
57 \\
225 \\
380 \\
376 \\
671 \\
717 \\
1456 \\
2367\end{array}$ & $\begin{array}{l}-5.92 \\
-5.58 \\
-5.16 \\
-4.75 \\
-4.24 \\
-3.92 \\
-3.50 \\
-3.07 \\
-2.72 \\
-2.37\end{array}$ & $\begin{array}{l}-7.30 \\
-6.95 \\
-6.50 \\
-6.06 \\
-5.51 \\
-5.15 \\
-4.70 \\
-4.21 \\
-3.86 \\
-3.48\end{array}$ & $\begin{array}{r}66 \\
35 \\
64 \\
120 \\
218 \\
215 \\
444 \\
792 \\
921 \\
\text { NaN }\end{array}$ \\
\hline $\begin{array}{l}\text { NGC } \\
7.4 \\
7.5 \\
7.6 \\
7.7 \\
7.8 \\
7.9 \\
8.0 \\
8.1\end{array}$ & $\begin{array}{c}5253, \mathrm{~V}= \\
-6.20 \\
-6.07 \\
-5.71 \\
-5.05 \\
-4.60 \\
-4.21 \\
-3.93 \\
-3.50\end{array}$ & $\begin{array}{r}F 555 W \\
-6.22 \\
-6.10 \\
-5.79 \\
-5.08 \\
-4.62 \\
-4.25 \\
-4.01 \\
-3.61\end{array}$ & $\begin{array}{r}206 \\
33 \\
118 \\
392 \\
381 \\
493 \\
443 \\
957\end{array}$ & $\begin{array}{l}-5.75 \\
-5.37 \\
-5.00 \\
-4.61 \\
-4.27 \\
-3.81 \\
-3.36 \\
-3.00\end{array}$ & $\begin{array}{l}-7.24 \\
-6.88 \\
-6.43 \\
-6.02 \\
-5.63 \\
-5.13 \\
-4.64 \\
-4.26\end{array}$ & $\begin{array}{r}169 \\
103 \\
116 \\
170 \\
212 \\
467 \\
\text { NaN } \\
\text { NaN }\end{array}$ \\
\hline $\begin{array}{l}\text { NGC } \\
7.4 \\
7.5 \\
7.6 \\
7.7 \\
7.8 \\
7.9 \\
8.0 \\
8.1 \\
8.2 \\
8.3 \\
8.4 \\
8.5 \\
8.6 \\
8.7\end{array}$ & $\begin{array}{l}6822(1), \\
-6.07 \\
-5.99 \\
-5.64 \\
-5.03 \\
-4.58 \\
-4.23 \\
-3.81 \\
-3.39 \\
-2.99 \\
-2.51 \\
-2.04 \\
-1.44 \\
-0.96 \\
-0.52\end{array}$ & $\begin{array}{c}\mathrm{V}=\mathrm{F} 555 \mathrm{~W} \\
-6.20 \\
-6.15 \\
-5.79 \\
-5.18 \\
-4.72 \\
-4.37 \\
-3.97 \\
-3.61 \\
-3.29 \\
-3.03 \\
-2.72 \\
-2.26 \\
-1.84 \\
-1.45\end{array}$ & $\begin{array}{r}0 \\
0 \\
0 \\
0 \\
0 \\
2 \\
16 \\
15 \\
19 \\
28 \\
30 \\
48 \\
51 \\
71\end{array}$ & $\begin{array}{l}-5.81 \\
-5.45 \\
-5.10 \\
-4.71 \\
-4.24 \\
-3.81 \\
-3.47 \\
-3.10 \\
-2.62 \\
-2.34 \\
-2.06 \\
-1.70 \\
-1.41 \\
-1.04\end{array}$ & $\begin{array}{l}-7.31 \\
-6.95 \\
-6.54 \\
-6.11 \\
-5.62 \\
-5.15 \\
-4.79 \\
-4.38 \\
-3.87 \\
-3.57 \\
-3.27 \\
-2.90 \\
-2.60 \\
-2.23\end{array}$ & $\begin{array}{r}0 \\
0 \\
0 \\
0 \\
0 \\
4 \\
9 \\
15 \\
24 \\
18 \\
35 \\
61 \\
100 \\
134\end{array}$ \\
\hline $\begin{array}{l}\mathrm{NG} \\
7.4 \\
7.5 \\
7.6 \\
7.7\end{array}$ & $\begin{array}{l}6822(2), \\
-6.10 \\
-6.02 \\
-5.62 \\
-5.05\end{array}$ & $\begin{array}{l}\mathrm{V}=\mathrm{F} 555 \mathrm{~W} \\
-6.24 \\
-6.17 \\
-5.80 \\
-5.21\end{array}$ & $\begin{array}{l}0 \\
0 \\
0 \\
0\end{array}$ & $\begin{array}{l}-5.85 \\
-5.50 \\
-5.05 \\
-4.68\end{array}$ & $\begin{array}{l}-7.35 \\
-6.99 \\
-6.50 \\
-6.08\end{array}$ & $\begin{array}{l}0 \\
0 \\
0 \\
0\end{array}$ \\
\hline
\end{tabular}


TABLE 3 - Continued

\begin{tabular}{|c|c|c|c|c|c|c|}
\hline $\begin{array}{l}\text { Log } \\
\text { Age }\end{array}$ & $\begin{array}{c}M_{V} \\
(\mathrm{mag})\end{array}$ & $\begin{array}{c}M_{F 814 W} \\
(\mathrm{mag})\end{array}$ & $\begin{array}{l}\text { No. BHeB } \\
\text { Stars }\end{array}$ & $\begin{array}{c}M_{V} \\
(\mathrm{mag})\end{array}$ & $\begin{array}{c}M_{F 814 W} \\
\text { (mag) }\end{array}$ & $\begin{array}{l}\text { No. RHeB } \\
\text { Stars }\end{array}$ \\
\hline 7.8 & -4.58 & -4.73 & 1 & -4.35 & -5.73 & 0 \\
\hline 7.9 & -4.18 & -4.33 & 5 & -3.86 & -5.21 & 0 \\
\hline 8.0 & -3.82 & -4.00 & 16 & -3.45 & -4.76 & 15 \\
\hline 8.1 & -3.48 & -3.71 & 18 & -3.03 & -4.29 & 30 \\
\hline 8.2 & -3.02 & -3.37 & 14 & -2.68 & -3.89 & 44 \\
\hline 8.3 & -2.51 & -3.04 & 34 & -2.34 & -3.55 & 48 \\
\hline 8.4 & -2.00 & -2.69 & 56 & -2.04 & -3.24 & 59 \\
\hline 8.5 & -1.49 & -2.31 & 90 & -1.69 & -2.86 & 148 \\
\hline 8.6 & -1.00 & -1.90 & 141 & -1.38 & -2.55 & 218 \\
\hline 8.7 & -0.48 & -1.40 & 261 & -1.13 & -2.30 & 158 \\
\hline \multicolumn{7}{|c|}{ NGC $6822(3),{ }^{\mathrm{a}} \mathrm{V}=\mathrm{F} 555 \mathrm{~W}$} \\
\hline 7.4 & -6.16 & -6.26 & 0 & -5.85 & -7.33 & 0 \\
\hline 7.5 & -6.02 & -6.17 & 0 & -5.45 & -6.91 & 0 \\
\hline 7.6 & -5.70 & -5.85 & 0 & -5.06 & -6.47 & 0 \\
\hline 7.7 & -5.02 & -5.11 & 0 & -4.69 & -6.06 & 0 \\
\hline 7.8 & -4.58 & -4.68 & 0 & -4.27 & -5.60 & 0 \\
\hline 7.9 & -4.27 & -4.38 & 0 & -3.87 & -5.19 & 0 \\
\hline 8.0 & -3.87 & -4.01 & 2 & -3.45 & -4.75 & 2 \\
\hline 8.1 & -3.45 & -3.65 & 3 & -3.13 & -4.38 & 4 \\
\hline 8.2 & -3.05 & -3.36 & 6 & -2.72 & -3.92 & 9 \\
\hline 8.3 & -2.52 & -2.99 & 13 & -2.35 & -3.53 & 19 \\
\hline 8.4 & -1.92 & -2.56 & 20 & -1.98 & -3.13 & 23 \\
\hline 8.5 & -1.49 & -2.25 & 11 & -1.70 & -2.83 & 29 \\
\hline 8.6 & -1.05 & -1.91 & 15 & -1.39 & -2.55 & 65 \\
\hline 8.7 & -0.48 & -1.35 & 22 & -1.11 & -2.27 & 40 \\
\hline \multicolumn{7}{|c|}{ NGC $4214, V=F 555 W$} \\
\hline 7.4 & -6.13 & -6.15 & 115 & -5.51 & -7.12 & 68 \\
\hline 7.5 & -5.93 & -5.98 & 26 & -5.14 & -6.72 & 84 \\
\hline 7.6 & -5.50 & -5.53 & 95 & -4.79 & -6.30 & 112 \\
\hline 7.7 & -5.06 & -5.10 & 134 & -4.46 & -5.92 & 109 \\
\hline 7.8 & -4.69 & -4.75 & 189 & -4.07 & -5.46 & 208 \\
\hline 7.9 & -4.27 & -4.39 & 323 & -3.76 & -5.12 & 249 \\
\hline 8.0 & -3.82 & -4.05 & 517 & -3.42 & -4.74 & 460 \\
\hline 8.1 & -3.28 & -3.69 & 756 & -2.94 & -4.21 & $\mathrm{NaN}$ \\
\hline \multicolumn{7}{|c|}{ NGC $4449, V=F 555 W$} \\
\hline 7.4 & -6.04 & -6.03 & 1009 & -5.54 & -7.19 & 581 \\
\hline 7.5 & -5.84 & -5.86 & 251 & -5.16 & -6.80 & 513 \\
\hline 7.6 & -5.47 & -5.50 & 638 & -4.82 & -6.39 & 616 \\
\hline 7.7 & -5.02 & -5.03 & 1261 & -4.52 & -6.04 & 665 \\
\hline 7.8 & -4.71 & -4.75 & 1348 & -4.18 & -5.67 & 1058 \\
\hline 7.9 & -4.30 & -4.38 & 2443 & -3.75 & -5.17 & 2363 \\
\hline 8.0 & -3.87 & -4.02 & 3858 & -3.41 & -4.81 & 3209 \\
\hline 8.1 & -3.47 & -3.79 & 5072 & -2.97 & -4.31 & $\mathrm{NaN}$ \\
\hline
\end{tabular}

NoTE. - The number of BHeB and RHeB stars per V-band and I-band luminosity bin and the associated age derived from isochrones. The V-band filters for each galaxy are listed next to the galaxy names; the I-band filter is F $814 \mathrm{~W}$ for all galaxies. NaN is listed for luminosities reaching below the photometric limit one can unambiguously separate blue or red $\mathrm{HeB}$ stars.

a The observations for NGC 6822 covered three fields of view. 